\title{
Stability of networks under level-k farsightedness
}

Citation for published version (APA):

Herings, P. J. J., Mauleon, A., \& Vannetelbosch, V. (2014). Stability of networks under level-k farsightedness. Maastricht University, Graduate School of Business and Economics. GSBE Research Memoranda No. 030 https://doi.org/10.26481/umagsb.2014030

Document status and date:

Published: 01/01/2014

DOI:

10.26481/umagsb.2014030

Document Version:

Publisher's PDF, also known as Version of record

\section{Please check the document version of this publication:}

- A submitted manuscript is the version of the article upon submission and before peer-review. There can be important differences between the submitted version and the official published version of record.

People interested in the research are advised to contact the author for the final version of the publication, or visit the DOI to the publisher's website.

- The final author version and the galley proof are versions of the publication after peer review.

- The final published version features the final layout of the paper including the volume, issue and page numbers.

Link to publication

\footnotetext{
General rights rights.

- You may freely distribute the URL identifying the publication in the public portal. please follow below link for the End User Agreement:

www.umlib.nl/taverne-license

Take down policy

If you believe that this document breaches copyright please contact us at:

repository@maastrichtuniversity.nl

providing details and we will investigate your claim.
}

Copyright and moral rights for the publications made accessible in the public portal are retained by the authors and/or other copyright owners and it is a condition of accessing publications that users recognise and abide by the legal requirements associated with these

- Users may download and print one copy of any publication from the public portal for the purpose of private study or research.

- You may not further distribute the material or use it for any profit-making activity or commercial gain

If the publication is distributed under the terms of Article $25 \mathrm{fa}$ of the Dutch Copyright Act, indicated by the "Taverne" license above, 


\section{Maastricht University}

P. Jean-Jacques Herings,

Ana Mauleon,

Vincent Vannetelbosch

Stability of Networks under Level-K Farsightedness

$\mathrm{RM} / 14 / 030$

\section{GSBE}

Maastricht University School of Business and Economics

Graduate School of Business and Economics

P.O Box 616

NL- 6200 MD Maastricht

The Netherlands 


\title{
Stability of Networks under Level- $K$ Farsightedness
}

\author{
P. Jean-Jacques Herings* Ana Mauleon ${ }^{\dagger}$ \\ Vincent Vannetelbosch ${ }^{\ddagger}$
}

July 10, 2014

\begin{abstract}
We provide a tractable concept that can be used to study the influence of the degree of farsightedness on network stability. A set of networks $G_{K}$ is a level- $K$ farsightedly stable set if three conditions are satisfied. First, external deviations should be deterred. Second, from any network outside of $G_{K}$ there is a sequence of farsighted improving paths of length smaller than or equal to $K$ leading to some network in $G_{K}$. Third, there is no proper subset of $G_{K}$ satisfying the first two conditions.

We show that a level- $K$ farsightedly stable set always exists and we provide a sufficient condition for the uniqueness of a level- $K$ farsightedly stable set. There is a unique level- 1 farsightedly stable set $G_{1}$ consisting of all networks that belong to closed cycles. Level- $K$ farsighted stability leads to a refinement of $G_{1}$ for generic allocation rules. We then provide easy to verify conditions for a set to be level- $K$ farsightedly stable and we consider the relationship between level- $K$ farsighted stability and efficiency of networks. We show the tractability of the concept by applying it to a model of criminal networks.
\end{abstract}

Key words: Farsightedness, Stability, Networks.

JEL classification: A14; C70; D20.

${ }^{*}$ Department of Economics, Maastricht University, Maastricht, The Netherlands. E-mail: P.Herings@maastrichtuniversity.nl

${ }^{\dagger}$ CEREC, Saint-Louis University - Brussels; CORE, University of Louvain, Louvain-la-Neuve, Belgium. E-mail: ana.mauleon@usaintlouis.be

${ }^{\ddagger}$ CORE, University of Louvain, Louvain-la-Neuve; CEREC, Saint-Louis University - Brussels, Belgium. E-mail: vincent.vannetelbosch@uclouvain.be 


\section{Introduction}

Networks of relationships help determine the careers that people choose, the jobs they obtain, the products they buy, and how they vote. The many aspects of our lives that are governed by social networks make it critical to understand how they impact behavior and which network structures are likely to emerge in a society. A simple way to analyze the networks that one might expect to emerge in the long run is to examine the requirement that individuals do not benefit from altering the structure of the network.

A prominent example of such a condition is the pairwise stability notion defined by Jackson and Wolinsky (1996). ${ }^{1}$ A network is pairwise stable if no individual benefits from deleting a link and no two individuals benefit from adding a link between them, with at least one benefitting strictly. While pairwise stability is natural, easy to work with and a very important tool in network analysis, ${ }^{2}$ it assumes that individuals are myopic, and not farsighted, in the sense that they do not forecast how others might react to their actions. Indeed, the addition or deletion of one link might lead to subsequent additions or deletions of other links. For instance, individuals might not add a link that appears valuable to them given the current network, as this might induce the formation of other links, ultimately leading to lower payoffs for them.

Herings, Mauleon and Vannetelbosch (2009) introduce the notion of pairwise farsighted stability. A set of networks is pairwise farsightedly stable (i) if all possible farsighted pairwise deviations from any network within the set to a network outside the set are deterred by the threat of ending worse off or equally well off, (ii) if there exists a farsighted improving path from any network outside the set leading to some network in the set, and (iii) if there is no proper subset satisfying conditions (i) and (ii). ${ }^{3}$ Pairwise farsighted stability makes sense if players have perfect anticipation

\footnotetext{
${ }^{1}$ An alternative way to model network stability is to explicitly model a game by which links form and then to solve that game using the concept of Nash equilibrium or one of its refinements. See Aumann and Myerson (1988), Myerson (1991) and Dutta and Mutuswami (1997) among others.

${ }^{2}$ Krishnan and Sciubba (2009) find that pairwise stability leads to testable predictions for the network architectures generated by labor-sharing groups in village economies of rural Ethiopia. In addition, their empirical results confirm strongly that the architecture of a social network and not just the number of links, has an important role to play in understanding network formation, and the role of social networks on economic performance.

${ }^{3}$ Other approaches to farsightedness in network formation are suggested by the work of Chwe
} 
of how others might react to changes in the network. But in general, especially when the set of players becomes large, it requires too much foresight on behalf of the players. ${ }^{4}$

Our aim is to provide a tractable concept that can be used to study the influence of the degree of farsightedness on network stability. We define the notion of a level$K$ farsightedly stable set. A set of networks $G_{K}$ is a level- $K$ farsightedly stable set if three conditions are satisfied. First, external deviations should be deterred. That is, adding a link $i j$ to a network $g \in G_{K}$ that leads to a network outside of $G_{K}$, is deterred by the threat of ending in $g^{\prime}$. Here $g^{\prime}$ is such that either there is a farsighted improving path of length smaller than or equal to $K-2$ from $g+i j$ to $g^{\prime}$ and $g^{\prime}$ belongs to $G_{K}$ or there is a farsighted improving path of length equal to $K-1$ from $g+i j$ to $g^{\prime}$ and there is no farsighted improving path from $g+i j$ to $g^{\prime}$ of smaller length. A similar requirement is imposed for the case where a link is severed. Second, external stability is required or, in other words, the networks within the set should be robust to perturbations. That is, from any network outside of $G_{K}$ there is a sequence of farsighted improving paths of length smaller than or equal to $K$ leading to some network in $G_{K}$. Third, a minimality condition is required. That is, there is no proper subset of $G_{K}$ satisfying the first two conditions.

We show that a level- $K$ farsightedly stable set always exists and we provide a sufficient condition for the uniqueness of a level- $K$ farsightedly stable set. We find that there is a unique level-1 farsightedly stable set $G_{1}$. It is given by the set consisting of all networks that belong to closed cycles, so it includes all pairwise stable networks. Level- $K$ farsighted stability leads to a refinement of myopic stability for generic allocation rules: for any $K \geq 1$, the myopically stable set $G_{1}$ contains a level- $K$ farsightedly stable set $G_{K}$. Thus, an analysis based on myopic behavior may not rule out some networks that are not stable when players are sufficiently farsighted. At the same time, a myopic analysis is compatible with farsightedness, and for any value of $K$ there is always a level- $K$ farsightedly stable set that consists (1994), Xue (1998), Herings, Mauleon and Vannetelbosch (2004), Mauleon and Vannetelbosch (2004), Dutta, Ghosal and Ray (2005), Page, Wooders and Kamat (2005), Page and Wooders (2009), Mauleon, Vannetelbosch and Vergote (2011), and Ray and Vohra (2013).

${ }^{4}$ Kirchsteiger, Mantovani, Mauleon and Vannetelbosch (2013) design a simple network formation experiment to test between pairwise stability and farsighted stability, but find evidence against both of them. Their experimental evidence suggests that subjects are consistent with an intermediate rule of behavior, which can be interpreted as a form of limited farsightedness. 
exclusively of networks that belong to closed cycles. But, some networks that are not part of any closed cycle may constitute a level- $K$ farsightedly stable set when $K$ strictly exceeds one.

We provide easy to verify conditions for a set to be level- $K$ farsightedly stable. We also consider the relationship between level- $K$ farsighted stability and efficiency of networks. We show that if there is a network that Pareto dominates all other networks, then that network is the unique prediction of level- $K$ farsighted stability if $K$ is greater than the maximum number of links in a network. In addition, we introduce a property on the allocation rule under which level- $K$ farsighted stability singles out the complete network. Finally, we illustrate the tractability of our new concept by analyzing the criminal network model of Calvo-Armengol and Zenou (2004). We find that in criminal networks with $n$ players, the set consisting of the complete network, so all criminals are linked to each other, is the unique level- $(n-1)$ farsightedly stable set.

Recent experimental and empirical studies suggest that players's initial choices in games often deviate systematically from equilibrium, that structural non-equilibrium level- $k$ (Stahl and Wilson, 1994; Nagel, 1995; Costa-Gomes, Crawford and Broseta, 2001) or cognitive hierarchy (Camerer, Ho and Chong, 2004) models often outpredict equilibrium, ${ }^{5}$ and that players only look a finite number of steps ahead when making choices. ${ }^{6}$ We assume that players are limited farsighted, but we do not require that players choose best responses to some beliefs on opponents' strategies. In our concept, players cannot even think about a strategy since they are not able to reason about what takes place after a certain reasoning horizon.

Recently, Morbitzer, Buskens and Rosenkranz (2011) develop a model of network formation where players look a finite number of steps ahead when anticipating the reaction of other players to their change. The decision to initiate a change to the

\footnotetext{
${ }^{5}$ Level- $k$ theory and the closely related cognitive hierarchy theory distinguish types of players according to the level at which they reason. Assumptions about level-0 behavior provide an anchor for beliefs and strategies at higher levels. At each higher level, players are assumed to know the probability distributions of strategies at lower levels. Level-1 players choose best responses to level-0 choices, while level- 2 players choose best responses to level- 1 choices (level- $k$ theory) or to some probability distribution over level-0 and level-1 strategies (cognitive hierarchy theory). See Crawford, Costa-Gomes and Iriberri (2013) for a review of the literature.

${ }^{6}$ Players who are motivated by substantial incentives often violate backward induction even in simple sequential games such as the centipede game. See McKelvey and Palfrey (1992) among others.
} 
network is based on some ad hoc rules that weigh improving paths that might follow their change, but which are not necessarily improving paths for the players who made the initial change. Using computer simulations they show that, in the co-author model of Jackson and Wolinsky (1996), limited farsighted players can overcome the tension between stability and efficiency only if the number of players is small. Their concept is a refinement of the set of pairwise stable networks and may therefore fail to exist.

The paper is organized as follows. In Section 2 we introduce some notations and basic properties and definitions for networks. In Section 3 we define the notions of improving paths and level- $K$ pairwise stability and we show that level- $K$ pairwise stable networks may fail to exist. In Section 4 we define the notion of a level- $K$ farsightedly stable set and we characterize it. In Section 5 we study the relationship to pairwise stability. In Section 6 we provide easy to verify sufficient conditions for a set to be level- $K$ farsightedly stable. We look at the relationship between level$K$ farsighted stability and efficiency of networks in Section 7. In that section we also show that under increasing returns to link creation, level- $K$ farsighted stability singles out the complete network. In Section 8 we analyze the Calvo-Armengol and Zenou (2004) model of criminal networks using level- $K$ farsighted stability. Finally, in Section 9 we conclude.

\section{Networks}

Let $N=\{1, \ldots, n\}$ be the finite set of players who are involved in some network relationship. The network relationships are reciprocal and the network is thus modeled as a non-directed graph. Individuals are the nodes in the graph and links indicate bilateral relationships between individuals. Thus, a network $g$ is simply a list of which pairs of individuals are linked to each other. We write $i j \in g$ to indicate that $i$ and $j$ are linked under the network $g$. The complete network on the set of players $S \subseteq N$ is denoted $g^{S}$ and is equal to the set of all subsets of $S$ of size $2 .{ }^{7}$ It follows in particular that the empty network is denoted by $g^{\emptyset}$. The set of all possible networks or graphs on $N$ is denoted by $\mathbb{G}$ and consists of all subsets of $g^{N}$. The cardinality of $\mathbb{G}$ is denoted by $n^{\prime}=2^{n(n-1) / 2}$.

\footnotetext{
${ }^{7}$ Throughout the paper we use the notation $\subseteq$ for weak inclusion and $\varsubsetneqq$ for strict inclusion. Finally, \# will refer to the notion of cardinality.
} 
The network obtained by adding link $i j$ to an existing network $g$ is denoted by $g+i j$ and the network that results from deleting link $i j$ from an existing network $g$ by $g-i j$. Let

$$
g_{\mid S}=\{i j \in g \mid i, j \in S\} .
$$

Thus, $g_{\mid S}$ is the network found by deleting all links except those that are between players in $S$. For any network $g$, let $N(g)=\{i \in N \mid \exists j \in N$ such that $i j \in g\}$ be the set of players who have at least one link in the network $g$.

A path in a network $g \in \mathbb{G}$ between players $i$ and $j$ of length $K \geq 1$ is a finite sequence of players $i_{0}, \ldots, i_{K}$ with $i_{0}=i$ and $i_{K}=j$ such that for any $k \in\{0, \ldots, K-1\}, i_{k} i_{k+1} \in g$, and such that each player in the sequence $i_{0}, \ldots, i_{K}$ is distinct. A network $g$ is connected if for each pair of players $i$ and $j$ in $N(g)$ such that $i \neq j$ there exists a path between $i$ and $j$ in $g$. This definition implies that the empty network $g^{\emptyset}$ is connected. A non-empty network $h \subseteq g$ is a component of $g$ if for all $i \in N(h)$ and $j \in N(h) \backslash\{i\}$, there exists a path in $h$ connecting $i$ and $j$, and for any $i \in N(h)$ and $j \in N(g), i j \in g$ implies $i j \in h$. The set of components of $g$ is denoted by $C(g)$. Using the components of a network, we can partition the players into maximal groups within which players are connected. Let $P(g)$ denote the partition of $N$ induced by the network $g$. That is, the set of player $S$ belongs to $P(g)$ if and only if either there exists a network $h$ in $C(g)$ such that $S=N(h)$ or there exists $i \notin N(g)$ such that $S=\{i\}$.

An allocation rule is a function $Y: \mathbb{G} \rightarrow \mathbb{R}^{N}$ which gives for every player $i$ and network $g$ a payoff $Y_{i}(g)$.

\section{Improving Paths}

A farsighted improving path of length $K \geq 0$ from a network $g$ to a network $g^{\prime} \neq g$ is a finite sequence of networks $g_{0}, \ldots, g_{K}$ with $g_{0}=g$ and $g_{K}=g^{\prime}$ such that for any $k \in\{0, \ldots, K-1\}$ either (i) $g_{k+1}=g_{k}-i j$ for some $i j$ such that $Y_{i}\left(g_{K}\right)>Y_{i}\left(g_{k}\right)$ or $Y_{j}\left(g_{K}\right)>Y_{j}\left(g_{k}\right)$, or (ii) $g_{k+1}=g_{k}+i j$ for some $i j$ such that $Y_{i}\left(g_{K}\right)>Y_{i}\left(g_{k}\right)$ and $Y_{j}\left(g_{K}\right) \geq Y_{j}\left(g_{k}\right)$. Since the set $\{0, \ldots, K-1\}$ is empty for $K=0$, this definition implies that there is a farsighted improving path of length 0 from each network $g$ to itself, but clearly there are no farsighted improving paths of length 0 from $g$ to any other network. If there exists a farsighted improving path of length $K$ from $g$ to $g^{\prime}$, then we write $g \rightarrow_{K} g^{\prime}$. 
For a given network $g$ and some $K^{\prime} \geq 0$, let $f_{K^{\prime}}(g)$ be the set of networks that can be reached from $g$ by a farsighted improving path of length $K \leq K^{\prime}$. That is,

$$
f_{K^{\prime}}(g)=\left\{g^{\prime} \in \mathbb{G} \mid \exists K \leq K^{\prime} \text { such that } g \rightarrow_{K} g^{\prime}\right\} .
$$

This defines $f_{K^{\prime}}$ as a correspondence on the set $\mathbb{G}$. The set of networks that can be reached from $g$ by some farsighted improving path is denoted by $f_{\infty}(g)$, so

$$
f_{\infty}(g)=\left\{g^{\prime} \in \mathbb{G} \mid \exists K \in \mathbb{N} \text { such that } g \rightarrow_{K} g^{\prime}\right\} .
$$

The following lemma follows almost immediately and is presented without proof.

Lemma 1. For $K \geq 0$, for every $g \in \mathbb{G}$, it holds that $f_{K}(g) \subseteq f_{K+1}(g)$. For $K \geq n^{\prime}-1$, for every $g \in \mathbb{G}$, it holds that $f_{K}(g)=f_{K+1}(g)=f_{\infty}(g)$.

For $K \geq 0$, we define the relation $\tilde{f}_{K}$ on $\mathbb{G}$ as $\tilde{f}_{K}(g)=f_{K}(g) \backslash\{g\}, g \in \mathbb{G}$, so the network $g$ is dropped from $f_{K}(g)$ and the set $\tilde{f}_{K}(g)$ corresponds to the networks different from $g$ that can be reached from $g$ by a farsighted improving path of length at most $K$ (and at least one). Similarly, we define $\tilde{f}_{\infty}$ by $\tilde{f}_{\infty}(g)=f_{\infty}(g) \backslash\{g\}$ for every $g \in \mathbb{G}$.

An important concept in the analysis of networks is the one of pairwise stability as introduced in Jackson and Wolinsky (1996).

Definition 1. A network $g \in \mathbb{G}$ is pairwise stable if

1. for every $i j \in g, Y_{i}(g) \geq Y_{i}(g-i j)$ and $Y_{j}(g) \geq Y_{j}(g-i j)$,

2. for every ij $\notin g$, if $Y_{i}(g)<Y_{i}(g+i j)$, then $Y_{j}(g)>Y_{j}(g+i j)$.

We say that a network $g^{\prime}$ is adjacent to $g$ if $g^{\prime}=g+i j$ or $g^{\prime}=g-i j$ for some $i j$. A network $g^{\prime}$ defeats $g$ if either $g^{\prime}=g-i j$ and $Y_{i}\left(g^{\prime}\right)>Y_{i}(g)$ or $Y_{j}\left(g^{\prime}\right)>Y_{j}(g)$, or if $g^{\prime}=g+i j$ with $\left(Y_{i}\left(g^{\prime}\right), Y_{j}\left(g^{\prime}\right)\right)>\left(Y_{i}(g), Y_{j}(g)\right){ }^{8}$ A network is pairwise stable if and only if it is not defeated by another network. ${ }^{9}$ It is also easy to see that $g^{\prime} \in \tilde{f}_{1}(g)$ if

\footnotetext{
${ }^{8}$ We use the notation $\left(Y_{i}\left(g^{\prime}\right), Y_{j}\left(g^{\prime}\right)\right)>\left(Y_{i}(g), Y_{j}(g)\right)$ for $Y_{i}\left(g^{\prime}\right) \geq Y_{i}(g)$ and $Y_{j}\left(g^{\prime}\right) \geq Y_{j}(g)$ with at least one inequality holding strictly, $\left(Y_{i}\left(g^{\prime}\right), Y_{j}\left(g^{\prime}\right)\right) \geq\left(Y_{i}(g), Y_{j}(g)\right)$ for $Y_{i}\left(g^{\prime}\right) \geq Y_{i}(g)$ and $Y_{j}\left(g^{\prime}\right) \geq Y_{j}(g)$, and $\left(Y_{i}\left(g^{\prime}\right), Y_{j}\left(g^{\prime}\right)\right) \gg\left(Y_{i}(g), Y_{j}(g)\right)$ for $Y_{i}\left(g^{\prime}\right)>Y_{i}(g)$ and $Y_{j}\left(g^{\prime}\right)>Y_{j}(g)$.

${ }^{9}$ Dutta and Mutuswami (1997) and Jackson and van den Nouweland (2005) introduce the notion of strong stability, where stability of the network against deviations by arbitrary coalitions is required. In the same spirit, our theory of limited farsightedness can easily be modified to study coalitional moves rather than pairwise moves.
} 
and only if $g^{\prime}$ defeats $g$. We can therefore characterize the pairwise stable networks as those $g \in \mathbb{G}$ for which $\tilde{f}_{1}(g)=\emptyset$, or, alternatively, $f_{1}(g)=\{g\}$. This formulation readily suggests the following stability notion when players are less myopic.

Definition 2. For $K \geq 1$, a network $g \in \mathbb{G}$ is level-K pairwise stable if $f_{K}(g)=\{g\}$.

We may replace the condition $f_{K}(g)=\{g\}$ for level- $K$ pairwise stability by the equivalent condition $\tilde{f}_{K}(g)=\emptyset$.

The set of level- $K$ pairwise stable networks is denoted by $P_{K}$. The set $P_{K}$ might be worth studying in its own right. However, similar to the case of myopic players, there is no guarantee that this set is non-empty. It follows from Lemma 1 that $P_{K} \supseteq P_{K+1}$, so emptiness is more likely to become a problem for higher values of $K{ }^{10}$ In the next section we present a stability notion that does not suffer from this emptiness problem.

The set $f_{K}^{2}(g)=f_{K}\left(f_{K}(g)\right)=\left\{g^{\prime \prime} \in \mathbb{G} \mid \exists g^{\prime} \in f_{K}(g)\right.$ such that $\left.g^{\prime \prime} \in f_{K}\left(g^{\prime}\right)\right\}$ consists of those networks that can be reached by a composition of two farsighted improving paths of length at most $K$ from $g$. We extend this definition and, for $m \in \mathbb{N}$, we define $f_{K}^{m}(g)$ as those networks that can be reached from $g$ by means of $m$ compositions of farsighted improving paths of length at most $K$. Since there are $n^{\prime}$ networks in $\mathbb{G}$, it follows that $f_{K}^{m}$ is the same for all values of $m$ greater than or equal to $n^{\prime}-1$. The resulting correspondence for such values of $m$ is called the transitive closure of $f_{K}$ and is denoted by $f_{K}^{\infty} \cdot 11$

Lemma 1 extends to compositions of $f_{K}$ and in particular to the transitive closure $f_{K}^{\infty}$ of $f_{K}$ as is shown in the following lemma, which is presented without proof.

Lemma 2. For $K \geq 0$, for every $g \in \mathbb{G}$, it holds that $f_{K}^{\infty}(g) \subseteq f_{K+1}^{\infty}(g)$. For $K \geq n^{\prime}-1$, for every $g \in \mathbb{G}$, it holds that $f_{K}^{\infty}(g)=f_{K+1}^{\infty}(g)=f_{\infty}^{\infty}(g)$.

Jackson and Watts (2002) have defined the notion of a closed cycle. A set of networks $C$ is a cycle if for any $g^{\prime} \in C$ and $g \in C \backslash\left\{g^{\prime}\right\}$, there exists a sequence

\footnotetext{
${ }^{10}$ Jackson (2008) defines a network to be farsightedly pairwise stable if there is no farsighted improving path emanating from it. This concept reverts to $P_{\infty}$ and refines the set of pairwise stable networks. A drawback of the definition is that it does not require that a farsighted improving path ends at a network that is stable itself. The set $P_{\infty}$ is similar to the farsighted core when only one link at a time can be deleted or added.

${ }^{11}$ Page and Wooders (2009) use the path dominance relation to define the notion of path dominance core. For some given $m \in \mathbb{N}$, a network $g$ path dominates $g^{\prime}$ if $g \in \widetilde{f}_{\infty}^{m}\left(g^{\prime}\right)$. The path dominance core contains all networks that are not path dominated, but it often fails to exist.
} 


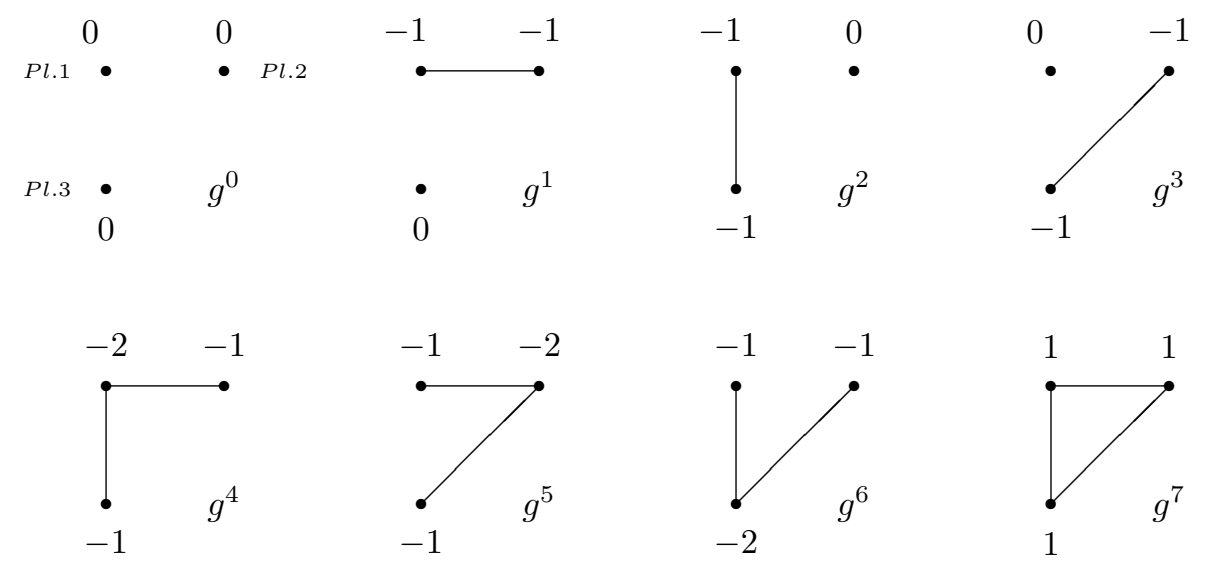

Figure 1: The 3-player investment networks.

of improving paths of length 1 connecting $g$ to $g^{\prime}$, i.e. $g^{\prime} \in f_{1}^{\infty}(g)$. A cycle $C$ is a maximal cycle if it is not a proper subset of a cycle. A cycle $C$ is a closed cycle if $f_{1}^{\infty}(C)=C$, so there is no sequence of improving paths of length 1 starting at some network in $C$ and leading to a network that is not in $C$. A closed cycle is necessarily a maximal cycle. For every network $g \in P_{1}$, the set $\{g\}$ is a closed cycle. The set of networks belonging to a closed cycle is non-empty.

We next present an example of an investment game as an example to illustrate the notion of farsighted improving paths and to point out some of its subtleties.

Example 1. The investment game - myopic analysis. Every player can have a link with another player at a cost of 1 . Every player receives a benefit of $n$ if all players have formed a link with all other players, but benefits are zero if at least one link is missing. Let $d_{i}(g)$ denote the number of links player $i$ has in $g$. Then it holds that $Y_{i}(g)=-d_{i}(g)$ if $g$ is not the complete network, and $Y_{i}\left(g^{N}\right)=n-d_{i}\left(g^{N}\right)=1$. Figure 1 presents the resulting payoffs for the case with 3 players.

We compute the farsighted improving paths of length $K=1$ from a given network $g$ to find the pairwise stable networks. It can easily be verified that

$$
\begin{array}{rlrl}
f_{1}(g) & =\left\{g^{\prime} \in \mathbb{G} \mid g^{\prime} \subseteq g, \#\left(g \backslash g^{\prime}\right) \leq 1\right\}, & & \# g \leq n(n-1) / 2-2, \\
f_{1}(g) & =\left\{g^{\prime} \in \mathbb{G} \mid g^{\prime} \subseteq g, \#\left(g \backslash g^{\prime}\right) \leq 1\right\} \cup\left\{g^{N}\right\}, & \# g=n(n-1) / 2-1, \\
f_{1}\left(g^{N}\right) & =\left\{g^{N}\right\} .
\end{array}
$$

In an investment game with three or more players, it holds that both the empty network and the complete network are pairwise stable, whereas there are no other pairwise stable networks. 
Next we consider the transitive closure of $f_{1}$ to compute the closed cycles in the investment game. It can easily be computed that

$$
\begin{aligned}
f_{1}^{\infty}(g) & =\left\{g^{\prime} \in \mathbb{G} \mid g^{\prime} \subseteq g\right\}, & & \# g \leq n(n-1) / 2-2, \\
f_{1}^{\infty}(g) & =\left\{g^{\prime} \in \mathbb{G} \mid g^{\prime} \subseteq g\right\} \cup\left\{g^{N}\right\}, & & \# g=n(n-1) / 2-1, \\
f_{1}^{\infty}\left(g^{N}\right) & =\left\{g^{N}\right\} . & &
\end{aligned}
$$

The empty network $g^{\emptyset}$ belongs to $f_{1}^{\infty}(g)$ for every $g$ that is not complete. Now it is not hard to verify that the closed cycles in the investment example coincide with the pairwise stable sets, so are given by the empty and the complete network.

It is a priori reasonable that the complete network is stable. However, this is less clear for the empty network and the question whether the empty network is stable or not should be intimately linked to the number of players and their degree of farsightedness. We continue the example by studying the farsighted improving paths of length $K \geq 2$.

Example 2. The investment game - farsighted analysis. When we consider farsighted improving paths of length $K=2$ or 3 , the complete network belongs to $f_{K}(g)$ if and only if $\#\left(g^{N} \backslash g\right) \leq K$, so the network $g^{N}$ can be obtained from $g$ by adding $K$ links. When the network $g$ is not complete, $f_{K}(g)$ also includes those networks that are obtained by deleting less than or equal to $K$ links from $g$, and no other networks.

The picture changes slightly when we consider farsighted paths of length 4 or higher. Although it is generally the case that $g^{N} \in f_{K}(g)$ if and only if $\#\left(g^{N} \backslash g\right) \leq$ $K$, and a subset $g^{\prime}$ of $g \neq g^{N}$ belongs to $f_{K}(g)$ if and only if $\#\left(g \backslash g^{\prime}\right) \leq K$, new possibilities arise. For instance, in a 4-player investment game it holds that $\{14,23\} \in$ $f_{4}(\{12,13,23,24\})$, so the link 14 , which did not exist in the starting network $\{12,13,23,24\}$, is added. Indeed, starting from the network $g=\{12,13,23,24\}$, first Players 1 and 4 form a link, next Player 1 severs his links with Players 2 and 3, and finally Player 2 cuts his link with Player 4. This constitutes a farsighted improving path since none of the networks involved in the path is complete, the degree of Player 1 in the network $\{12,13,23,24\}$ is one higher than in the network $\{14,23\}$ and the degree of Player 4 is the same, so the addition of the link 14 in the beginning is feasible. From then on, only links are deleted, which improves the payoffs of the players involved and does not affect the other players. 
Despite the subtleties for higher values of $K$, it is straightforward to verify that the set $P_{K}$ of level- $K$ pairwise stable sets consists of $g^{\emptyset}$ and $g^{N}$ when $n(n-1) / 2>K$ and is equal to $\left\{g^{N}\right\}$ otherwise. When the level of farsightedness of players is greater than or equal to $n(n-1) / 2$, the number of links needed to go from the empty network to the complete network, the complete network emerges as the unique level- $K$ pairwise stable set. The level- $K$ pairwise stable set thereby captures the desired relation between the degree of farsightedness and the instability of the empty network. In many examples, however, the set $P_{K}$ will be empty and can therefore not be used to analyze the relation between the degree of farsightedness and stability. It also suffers from the drawback that the networks to which players plan to deviate might not be level- $K$ pairwise stable themselves.

\section{Level- $K$ Farsighted Stability}

To analyze the influence of the degree of farsightedness on the stability of networks, we define the notion of a level- $K$ farsightedly stable set. In the next definition, we use the notational convention that $f_{-1}(g)=\emptyset$ for every $g \in \mathbb{G}$.

Definition 3. For $K \geq 1$, a set of networks $G_{K} \subseteq \mathbb{G}$ is a level- $K$ farsightedly stable set with respect to $Y$ if

(i) $\forall g \in G_{K}$,

(ia) $\forall i j \notin g$ such that $g+i j \notin G_{K}$,

$\exists g^{\prime} \in\left[f_{K-2}(g+i j) \cap G_{K}\right] \cup\left[f_{K-1}(g+i j) \backslash f_{K-2}(g+i j)\right]$ such that $\left(Y_{i}\left(g^{\prime}\right), Y_{j}\left(g^{\prime}\right)\right)=\left(Y_{i}(g), Y_{j}(g)\right)$ or $Y_{i}\left(g^{\prime}\right)<Y_{i}(g)$ or $Y_{j}\left(g^{\prime}\right)<Y_{j}(g)$,

(ib) $\forall i j \in g$ such that $g-i j \notin G_{K}$,

$\exists g^{\prime}, g^{\prime \prime} \in\left[f_{K-2}(g-i j) \cap G_{K}\right] \cup\left[f_{K-1}(g-i j) \backslash f_{K-2}(g-i j)\right]$ such that $Y_{i}\left(g^{\prime}\right) \leq Y_{i}(g)$ and $Y_{j}\left(g^{\prime \prime}\right) \leq Y_{j}(g)$.

(ii) $\forall g^{\prime} \in \mathbb{G} \backslash G_{K}, f_{K}^{\infty}\left(g^{\prime}\right) \cap G_{K} \neq \emptyset$.

(iii) $\forall G_{K}^{\prime} \varsubsetneqq G_{K}$, at least one of the Conditions (ia), (ib), and (ii) is violated by $G_{K}^{\prime}$.

The move from a network $g$ to an adjacent network is called a deviation. Condition (i) in Definition 3 requires the deterrence of external deviations. Condition (ia) 
captures that adding a link $i j$ to a network $g \in G_{K}$ that leads to a network outside of $G_{K}$, is deterred by the threat of ending in $g^{\prime}$. Here $g^{\prime}$ is such that either there is a farsighted improving path of length smaller than or equal to $K-2$ from $g+i j$ to $g^{\prime}$ and $g^{\prime}$ belongs to $G_{K}$ or there is a farsighted improving path of length equal to $K-1$ from $g+i j$ to $g^{\prime}$ and there is no farsighted improving path from $g+i j$ to $g^{\prime}$ of smaller length. Condition (ib) is a similar requirement, but then for the case where a link is severed. ${ }^{12}$

Since level- $K$ farsightedness models a reasoning horizon of the players of length $K$, we have to distinguish farsighted improving paths of length less than or equal to $K-2$ after a deviation from $g$ to $g+i j$ and farsighted improving paths of length equal to $K-1$. In the former case, the reasoning capacity of the players is not yet reached, and the threat of ending in $g^{\prime}$ is only credible if it belongs to the farsightedly stable set $G_{K}$. In the latter case, the only way to reach $g^{\prime}$ from $g$ requires $K$ steps of reasoning or even more; one step in the deviation to $g+i j$ and at least $K-1$ additional steps in any farsighted improving path from $g+i j$ to $g^{\prime}$. Since this exhausts the reasoning capacity of the players, the threat of ending in $g^{\prime}$ is credible, irrespective of whether it belongs to $G_{K}$ or not.

Condition (ii) in Definition 3 requires external stability and implies that the networks within the set are robust to perturbations. From any network outside of $G_{K}$ there is a a sequence of farsighted improving paths of length smaller than or equal to $K$ leading to some network in $G_{K} \cdot{ }^{13}$ Condition (ii) implies that if a set of networks is level- $K$ farsightedly stable, it is non-empty. Condition (iii) is the minimality condition.

Condition (i) in Definition 3 guarantees that networks inside the set $G_{K}$ are stable for players whose reasoning horizon is of length $K$. Hence, $f_{K}$ is used for deterring deviations from networks inside the set $G_{K}$. Condition (ii) in Definition 3 requires external stability and also implies robustness to perturbations of networks inside the set $G_{K}$. Perturbations may be due to exogenous forces acting on the

\footnotetext{
${ }^{12}$ Chwe (1994) defines the notion of largest consistent set. A set $G$ is a consistent set if both external and internal deviations with respect to $\widetilde{f}_{\infty}$ are deterred. The largest consistent is the set that contains any consistent set.

${ }^{13}$ Chwe (1994) defines the notion of von Neumann-Morgenstern farsightedly stable set. A set $G$ is a von Neumann-Morgenstern farsightedly stable set if both external and internal stability with respect to $\widetilde{f}_{\infty}$ are satisfied. Page and Wooders (2009) extends this notion by requiring both external and internal stability with respect to $\widetilde{f}_{\infty}^{m}$ for given $m \in \mathbb{N}$.
} 
network, or simply errors on the part of some players. When players make linking decisions at a network outside $G_{K}$, they move according to some level- $K$ farsighted improving path without being able to anticipate that other linking decisions might be taken afterwards. Hence, $f_{K}^{\infty}$ is used to capture what could happen at a network outside $G_{K}$. Condition (ii) implies that if we allow level- $K$ farsighted players to successively create or delete links, they will ultimately reach the set $G_{K}$ irrespective of the initial network.

Condition (ii) immediately implies that a level- $K$ farsightedly stable set of networks contains at least one network, so satisfies non-emptiness. The next result claims existence.

Theorem 1. A level-K farsightedly stable set of networks exists.

Proof. Notice that $\mathbb{G}$ trivially satisfies Conditions (i) and (ii). Let us proceed by contradiction. Assume that there does not exist any set of networks $G_{K} \subseteq \mathbb{G}$ that is level- $K$ farsightedly stable. This means that for any $G_{K}^{0} \subseteq \mathbb{G}$ that satisfies Conditions (i) and (ii) in Definition 3, we can find a proper subset $G_{K}^{1}$ that satisfies Conditions (i) and (ii). Iterating this reasoning, we can construct an infinite sequence $\left\{G_{K}^{k}\right\}_{k \geq 0}$ of subsets of $\mathbb{G}$ satisfying Conditions (i) and (ii) with the property that $G_{K}^{k} \subsetneq G_{K}^{k-1}$. But since $\mathbb{G}$ has finite cardinality $n^{\prime}$, this is not possible.

For the special case where $K$ is equal to 1 , we can use the fact that $f_{-1}(g)=\emptyset$ and $f_{0}(g)=\{g\}$, so Definition 3 simplifies as follows.

Theorem 2. A set of networks $G_{1} \subseteq \mathbb{G}$ is a level-1 farsightedly stable set with respect to $Y$ if

(i) $\forall g \in G_{1}$,

(ia) $\forall i j \notin g$ such that $g^{\prime}=g+i j \notin G_{1}$ it holds that $\left(Y_{i}\left(g^{\prime}\right), Y_{j}\left(g^{\prime}\right)\right)=$ $\left(Y_{i}(g), Y_{j}(g)\right)$ or $Y_{i}\left(g^{\prime}\right)<Y_{i}(g)$ or $Y_{j}\left(g^{\prime}\right)<Y_{j}(g)$,

(ib) $\forall i j \in g$ such that $g^{\prime}=g-i j \notin G_{1}$ it holds that $Y_{i}\left(g^{\prime}\right) \leq Y_{i}(g)$ and $Y_{j}\left(g^{\prime}\right) \leq Y_{j}(g)$.

(ii) $\forall g^{\prime} \in \mathbb{G} \backslash G_{1}, f_{1}^{\infty}\left(g^{\prime}\right) \cap G_{1} \neq \emptyset$.

(iii) $\forall G_{1}^{\prime} \varsubsetneqq G_{1}$, at least one of the Conditions (ia), (ib), and (ii) is violated by $G_{1}^{\prime}$. 
Theorem 2 shows that a level-1 farsightedly stable set is identical to a myopically stable set as defined in Herings, Mauleon and Vannetelbosch (2009). Herings, Mauleon and Vannetelbosch (2009) have shown that there is a unique myopically stable set. It is equal to the set of networks consisting of all networks that belong to a closed cycle. Theorem 3 below follows.

Theorem 3. There is a unique level-1 farsightedly stable set. It is given by the set consisting of all networks that belong to a closed cycle.

Since a farsightedly stable set cannot be empty, it follows from Theorem 3 that there is at least one closed cycle. Level-1 farsightedly stable sets are unique. This result does not carry over to higher levels of $K$.

Also for $K=2$, the definition of a level- $K$ farsightedly stable set simplifies somewhat, since if a network $g+i j$ belongs to $\mathbb{G} \backslash G_{2}$ for some set $G_{2}$, it holds that $f_{0}(g+i j) \cap G_{2}=\{g+i j\} \cap G_{2}=\emptyset$.

Theorem 4. A set of networks $G_{2} \subseteq \mathbb{G}$ is a level-2 farsightedly stable set with respect to $Y$ if

(i) $\forall g \in G_{2}$,

(ia) $\forall i j \notin g$ such that $g+i j \notin G_{2}, \exists g^{\prime} \in \tilde{f}_{1}(g+i j)$ such that $\left(Y_{i}\left(g^{\prime}\right), Y_{j}\left(g^{\prime}\right)\right)=$ $\left(Y_{i}(g), Y_{j}(g)\right)$ or $Y_{i}\left(g^{\prime}\right)<Y_{i}(g)$ or $Y_{j}\left(g^{\prime}\right)<Y_{j}(g)$,

(ib) $\forall i j \in g$ such that $g-i j \notin G_{2}, \exists g^{\prime}, g^{\prime \prime} \in \tilde{f}_{1}(g-i j)$ such that $Y_{i}\left(g^{\prime}\right) \leq Y_{i}(g)$ and $Y_{j}\left(g^{\prime \prime}\right) \leq Y_{j}(g)$.

(ii) $\forall g^{\prime} \in \mathbb{G} \backslash G_{2}, f_{2}^{\infty}\left(g^{\prime}\right) \cap G_{2} \neq \emptyset$.

(iii) $\forall G_{2}^{\prime} \varsubsetneqq G_{2}$, at least one of the Conditions (ia), (ib), and (ii) is violated by $G_{2}^{\prime}$.

Theorem 4 is useful when computing level-2 farsightedly stable sets in examples. At the other extreme, when $K$ is greater than or equal to $n^{\prime}+1$, it follows from Lemma 1 that $f_{K-2}(g)=f_{K-1}(g)$ for every $g \in \mathbb{G}$, and from Lemma 2 that $f_{K}^{\infty}(g)=$ $f_{n^{\prime}-1}^{\infty}(g)$ for every $g \in \mathbb{G}$. We therefore have the following result.

Theorem 5. For $K \geq n^{\prime}+1$, a set of networks $G_{K} \subseteq \mathbb{G}$ is a level-K farsightedly stable set with respect to $Y$ if

(i) $\forall g \in G_{K}$, 
(ia) $\forall i j \notin g$ such that $g+i j \notin G_{K}, \exists g^{\prime} \in f_{n^{\prime}-1}(g+i j) \cap G_{K}$ such that $\left(Y_{i}\left(g^{\prime}\right), Y_{j}\left(g^{\prime}\right)\right)=\left(Y_{i}(g), Y_{j}(g)\right)$ or $Y_{i}\left(g^{\prime}\right)<Y_{i}(g)$ or $Y_{j}\left(g^{\prime}\right)<Y_{j}(g)$,

(ib) $\forall i j \in g$ such that $g-i j \notin G_{K}, \exists g^{\prime}, g^{\prime \prime} \in f_{n^{\prime}-1}(g-i j) \cap G_{K}$ such that $Y_{i}\left(g^{\prime}\right) \leq Y_{i}(g)$ and $Y_{j}\left(g^{\prime \prime}\right) \leq Y_{j}(g)$.

(ii) $\forall g^{\prime} \in \mathbb{G} \backslash G_{K}, f_{n^{\prime}-1}^{\infty}\left(g^{\prime}\right) \cap G_{K} \neq \emptyset$.

(iii) $\forall G_{K}^{\prime} \varsubsetneqq G_{K}$, at least one of the Conditions (ia), (ib), and (ii) is violated by $G_{K}^{\prime}$

It follows immediately from Theorem 5 that the collection of $K$-farsightedly stable sets is independent of $K$ when $K \geq n^{\prime}+1$.

Herings, Mauleon and Vannetelbosch (2009) define a farsightedly stable set as a set $G_{\infty}$ of networks satisfying Conditions (i) and (iii) of Theorem 5, but with Condition (ii) replaced by the requirement that

$$
\forall g^{\prime} \in \mathbb{G} \backslash G_{\infty}, f_{\infty}\left(g^{\prime}\right) \cap G_{\infty} \neq \emptyset
$$

so the correspondence $f_{n^{\prime}-1}^{\infty}$ is replaced by $f_{\infty}=f_{n^{\prime}-1}$, and one could interpret the Herings, Mauleon and Vannetelbosch (2009) concept as level- $\infty$ farsighted stability. In many applications, the correspondence $f_{\infty}$ is transitive, in which case it coincides with $f_{n^{\prime}-1}^{\infty}$, and level- $\infty$ farsighted stable sets are identical to level- $\left(n^{\prime}+1\right)$ farsightedly stable sets, but in general it only holds that $f_{\infty}(g)=f_{n^{\prime}-1}(g) \subseteq f_{n^{\prime}-1}^{\infty}(g)$ for $g \in \mathbb{G}$. We can therefore conclude that for every level- $\infty$ farsightedly stable set $G_{\infty}$ there is a set $G^{\prime} \subseteq G_{\infty}$ such that $G^{\prime}$ is level- $\left(n^{\prime}+1\right)$ farsightedly stable.

Example 3. Investment game - farsightedly stable sets. We now analyze the concept of a level- $K$ farsightedly stable set for the investment game of Example 1. For level-1 farsightedly stable sets, we can use Theorem 3 and have to identify all the closed cycles. Using the analysis in Example 1, we find that the unique farsightedly stable set consists of the empty and the complete network whenever there are at least three players.

We will argue next that with $n \geq 3$ players, a reasoning horizon of length $K$ equal to $n(n-1) / 2$ or higher is needed to obtain the complete network as the unique level$K$ farsightedly stable set. For $K<n(n-1) / 2$, we show that the unique level- $K$ farsightedly stable set consists of the empty and the complete network. 
We argue first that $\left\{g^{N}\right\}$ is the unique level- $K$ farsightedly stable set when $K \geq n(n-1) / 2 \geq 3$. The analysis in Example 1 reveals that $g^{N} \in f_{1}(\bar{g})$ for all networks $\bar{g}$ that are adjacent to $g^{N}$, so by Lemma 1 we have $g^{N} \in f_{K-2}(\bar{g})$ for all networks $\bar{g}$ that are adjacent to $g^{N}$, and Condition (i) of Definition 3 is satisfied since a deviation from $g^{N}$ to an adjacent network $\bar{g}$ is deterred by the return to $g^{N}$.

We have argued in Example 2 that $g^{N} \in f_{K}\left(g^{\prime}\right)$ if and only if $\#\left(g^{N} \backslash g^{\prime}\right) \leq K$. It follows that $g^{N} \in f_{K}\left(g^{\prime}\right)$ for every $g^{\prime} \neq g^{N}$, since $\#\left(g^{N} \backslash g^{\prime}\right) \leq n(n-1) / 2 \leq$ $K$ for every $g^{\prime} \neq g^{N}$. Since $f_{K}(g) \subseteq f_{K}^{\infty}(g)$, we have for every $g^{\prime} \in \mathbb{G} \backslash\left\{g^{N}\right\}$, $f_{K}^{\infty}\left(g^{\prime}\right) \cap\left\{g^{N}\right\} \neq \emptyset$ and Condition (ii) of Definition 3 is satisfied. Obviously, $\left\{g^{N}\right\}$ satisfies minimality as expressed in Condition (iii) of Definition 3 , so $g^{N}$ is a level- $K$ farsightedly stable set.

Since $Y\left(g^{N}\right) \gg Y(g)$ for every $g \in \mathbb{G} \backslash\left\{g^{N}\right\}$, it holds that $f_{K}\left(g^{N}\right)=\left\{g^{N}\right\}$ for every value of $K \geq 1$, and so $f_{K}^{\infty}\left(g^{N}\right)=\left\{g^{N}\right\}$. By Condition (ii) of Definition 3, it follows that $g^{N} \in G_{K}$ for every level- $K$ farsightedly stable set $G_{K}$. Minimality as expressed by Condition (iii) of Definition 3 now implies that $\left\{g^{N}\right\}$ is the unique level- $K$ farsightedly stable set when $K \geq n(n-1) / 2$.

Consider next the case $K<n(n-1) / 2$. It holds that $g^{N} \notin f_{K}\left(g^{\emptyset}\right)$, since one needs to form $n(n-1) / 2$ links to go from the empty to the complete network. Since $Y\left(g^{\emptyset}\right) \geq Y(g)$ for every $g \in \mathbb{G} \backslash\left\{g^{N}\right\}$, it follows that $f_{K}\left(g^{\emptyset}\right)=\left\{g^{\emptyset}\right\}$. By Condition (ii) of Definition 3, it follows that $g^{\emptyset} \in G_{K}$ for every level- $K$ farsightedly stable set $G_{K}$. In the previous paragraph we have argued that $g^{N} \in G_{K}$. The analysis in Example 2 reveals that $f_{K}^{\infty}\left(g^{\prime}\right) \cap\left\{g^{\emptyset}, g^{N}\right\} \neq \emptyset$ for every $g^{\prime} \in \mathbb{G} \backslash\left\{g^{\emptyset}, g^{N}\right\}$. Together with Condition (iii) of Definition 3, we now find that $\left\{g^{\emptyset}, g^{N}\right\}$ is the unique level- $K$ farsightedly stable set when $K<n(n-1) / 2$.

\section{The Relation to Pairwise Stability}

In this section, we discuss how level- $K$ farsightedly stable sets are related to notions based on pairwise stability such as the set of pairwise stable networks $P_{1}$, the set of closed cycles $G_{1}$, and the set of level- $K$ pairwise stable networks $P_{K}$.

Theorem 3 implies that any pairwise stable network belongs to $G_{1}$. The following theorem shows that this result carries over to higher values of $K$.

Theorem 6. For $K \geq 1$, the set $P_{K}$ of level-K pairwise stable networks is a subset of any level-K farsightedly stable set $G_{K}$. 
Proof. Suppose $G_{K}$ is level- $K$ farsightedly stable, but does not contain some $g \in P_{K}$. By Definition 2, we have $f_{K}(g)=\{g\}$. We find that $f_{K}^{\infty}(g)=\{g\}$, so $f_{K}^{\infty}(g) \cap G_{K}=\emptyset$. By Condition (ii) of Definition 3, it holds that $f_{K}^{\infty}(g) \cap G_{K} \neq \emptyset$, a contradiction.

Theorem 6 shows that any network $g$ from which there are no farsighted improving paths of length smaller than or equal to $K$ to networks different from $g$ belongs to $G_{K}$. Level- $K$ pairwise stability is quite demanding for higher levels of $K$. Since $f_{K}(g) \subseteq f_{K+1}(g)$, we have that $P_{K} \supseteq P_{K+1}$.

Theorem 6 yields an easy sufficient condition for the uniqueness of a level- $K$ farsightedly stable set as a corollary, where we make use of the minimality requirement as expressed in Condition (iii) of Definition 3.

Corollary 1. For $K \geq 1$, if $P_{K}$ is a level- $K$ farsightedly stable set, then it is uniquely so.

An allocation rule is said to be generic if for every $g, g^{\prime} \in \mathbb{G}$ such that $g$ and $g^{\prime}$ are adjacent it holds that either $g \in f_{1}\left(g^{\prime}\right)$ or $g^{\prime} \in f_{1}(g)$. If an allocation rule is not generic, then some arbitrarily small perturbation of it will be, and genericity can therefore be thought of as a weak requirement on allocation rules. The next result shows that level- $K$ farsighted stability leads to a refinement of myopic stability for generic allocation rules.

Theorem 7. Let the allocation rule be generic. For every $K \geq 1$, the myopically stable set $G_{1}$ contains a level-K farsightedly stable set $G_{K}$.

Proof. The statement is trivial for $K=1$, so we consider $K \geq 2$.

We show first that the set $G_{1}$ satisfies Condition (i) of Definition 3. Consider some $g \in G_{1}$ and a deviation to $g^{\prime} \in \mathbb{G} \backslash G_{1}$.

Suppose that $g^{\prime} \in f_{1}(g)$. Since $g \in G_{1}$ and $G_{1}$ contains all networks in a closed cycle by Theorem 3 , it follows that $g^{\prime} \in G_{1}$, a contradiction to $g^{\prime} \in \mathbb{G} \backslash G_{1}$. Consequently, it holds that $g^{\prime} \notin f_{1}(g)$.

Since the allocation rule is generic, we find that $g \in f_{1}\left(g^{\prime}\right)$. We have that $g \in f_{1}\left(g^{\prime}\right) \backslash\left\{g^{\prime}\right\}$, so for $K=2$ the deviation from $g$ to $g^{\prime}$ is deterred by $g$. For $K \geq 3$, we have by Lemma 1 that $g \in f_{1}\left(g^{\prime}\right) \cap G_{1} \subseteq f_{K-2}\left(g^{\prime}\right) \cap G_{1}$, so again the deviation from $g$ to $g^{\prime}$ is deterred by $g$.

We show next that the set $G_{1}$ satisfies Condition (ii) of Definition 3. Since $G_{1}$ is level-1 farsightedly stable, it holds for every $g^{\prime} \in \mathbb{G} \backslash G_{1}$ that $f_{1}^{\infty}\left(g^{\prime}\right) \cap G_{1} \neq \emptyset$. By 


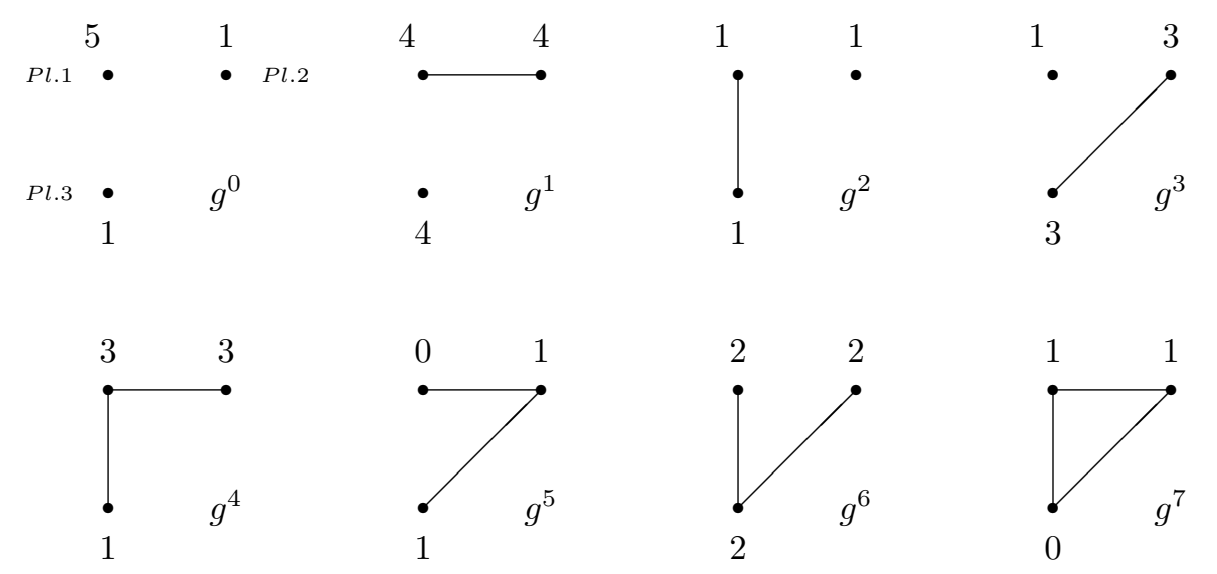

Figure 2: Networks outside closed cycles can be farsightedly stable in Example 4.

Lemma 2 it holds that $f_{1}^{\infty}\left(g^{\prime}\right) \subseteq f_{K}^{\infty}\left(g^{\prime}\right)$, so $f_{K}^{\infty}\left(g^{\prime}\right) \cap G_{1} \neq \emptyset$, and it follows that $G_{1}$ satisfies Condition (ii).

Either the set $G_{1}$ is a minimal set satisfying Conditions (i) and (ii) of Definition 3 and is therefore level- $K$ farsightedly stable, or it has a proper subset $G_{K}$ which is a minimal set satisfying Conditions (i) and (ii), so $G_{K}$ is level- $K$ farsightedly stable. In both cases, the statement of the theorem holds.

Theorem 3 asserts that there is a unique level- 1 farsightedly stable set $G_{1}$, given by the union of all closed cycles. Theorem 7 shows that higher levels of farsightedness lead to a refinement of the networks that belong to closed cycles. For any value of $K$, there is always a subset of $G_{1}$ that is level- $K$ farsightedly stable. Theorem 7 shows that an analysis based on myopic behavior may not rule out some networks that are not stable when players are sufficiently farsighted. At the same time, a myopic analysis is compatible with farsightedness, and for any value of $K$ there is always a farsightedly stable set that consists exclusively of networks that belong to closed cycles.

Theorem 7 does not claim that farsightedly stable sets are always subsets of networks in $G_{1}$. The following example shows that networks that are not part of any closed cycle may become stable for higher values of $K$.

Example 4. Consider the situation where three players can form and sever links and where the payoffs are given as in Figure 2. The farsighted improving paths of various lengths are presented in Table 1. 


\begin{tabular}{|c||c|c|c|c|}
\hline$g$ & $\tilde{f}_{1}(g)$ & $\tilde{f}_{2}(g)$ & $\tilde{f}_{3}(g)$ & $\tilde{f}_{K}(g), K \geq 4$ \\
\hline \hline$g^{0}$ & $g^{3}$ & $g^{3}$ & $g^{1}, g^{3}$ & $g^{1}, g^{3}, g^{4}$ \\
\hline$g^{1}$ & $g^{0}$ & $g^{0}$ & $g^{0}$ & $g^{0}$ \\
\hline$g^{2}$ & $g^{0}, g^{4}, g^{6}$ & $g^{0}, g^{1}, g^{3}, g^{4}, g^{6}$ & $g^{0}, g^{1}, g^{3}, g^{4}, g^{6}$ & $g^{0}, g^{1}, g^{3}, g^{4}, g^{6}$ \\
\hline$g^{3}$ & & $g^{1}$ & $g^{1}, g^{4}$ & $g^{1}, g^{4}$ \\
\hline$g^{4}$ & $g^{1}$ & $g^{0}, g^{1}$ & $g^{0}, g^{1}, g^{3}$ & $g^{0}, g^{1}, g^{3}$ \\
\hline$g^{5}$ & $g^{1}, g^{3}$ & $g^{1}, g^{3}, g^{4}, g^{6}$ & $g^{1}, g^{3}, g^{4}, g^{6}$ & $g^{0}, g^{1}, g^{3}, g^{4}, g^{6}$ \\
\hline$g^{6}$ & $g^{3}$ & $g^{3}, g^{4}$ & $g^{1}, g^{3}, g^{4}$ & $g^{1}, g^{3}, g^{4}$ \\
\hline$g^{7}$ & $g^{4}, g^{5}, g^{6}$ & $g^{1}, g^{3}, g^{4}, g^{5}, g^{6}$ & $g^{0}, g^{1}, g^{3}, g^{4}, g^{5}, g^{6}$ & $g^{0}, g^{1}, g^{3}, g^{4}, g^{5}, g^{6}$ \\
\hline
\end{tabular}

Table 1: The elements of $\tilde{f}_{K}(g)$ in Example 4 .

In this example there is a unique pairwise stable network, $g^{3}$. By inspecting $\tilde{f}_{1}$ as presented in Table 1, it is easily verified that there are no other closed cycles in this example, so $G_{1}=\left\{g^{3}\right\}$. By Theorem 7 , and the fact that each farsightedly stable set contains at least one element, it holds that $\left\{g^{3}\right\}$ is a level- $K$ farsightedly stable set for any value of $K$. At the same time, the payoffs resulting from the network $g^{3}$ are Pareto dominated by those of $g^{1}$. The problem with network $g^{1}$ is that Player 1 has myopic incentives to cut his link with Player 2 to obtain a payoff of 5 from the network $g^{0}$ instead of 4 from the network $g^{1}$. Once at $g^{0}$, Players 2 and 3 have myopic incentives to form a link and form the pairwise stable network $g^{3}$. The question is whether the Pareto efficient network $g^{1}$ is stable when the players are less myopic.

We first show that $\left\{g^{1}\right\}$ is a level-2 farsightedly stable set by verifying that $\left\{g^{1}\right\}$ satisfies the three conditions in Theorem 4. There are three possible deviations from $g^{1}$. Players 1 and 2 can cut their link and move to $g^{0}$, Players 1 and 3 can form a link to arrive at $g^{4}$, and Players 2 and 3 can form a link to go to $g^{5}$. From Table 1 it follows immediately that $g^{3} \in \tilde{f}_{1}\left(g^{0}\right), g^{1} \in \tilde{f}_{1}\left(g^{4}\right)$, and $g^{1} \in \tilde{f}_{1}\left(g^{5}\right)$. Since Players 1 and 2 both have lower payoffs at $g^{3}$ than at $g^{1}$, the first deviation is deterred. The other two deviations are deterred by the possible return to $g^{1}$. We conclude that Condition (i) of Theorem 4 holds.

One degree of farsightedness is needed to move from $g^{4}$ or $g^{5}$ to $g^{1}$, and two such degrees are needed to move from $g^{2}, g^{3}$, or $g^{7}$ to $g^{1}$. Since $g^{0} \rightarrow_{1} g^{3} \rightarrow_{2} g^{1}$ and $g^{6} \rightarrow_{1} g^{3} \rightarrow_{2} g^{1}$, we have shown that for every $g^{\prime} \in \mathbb{G} \backslash\left\{g^{1}\right\}, g^{1} \in f_{2}^{\infty}\left(g^{\prime}\right)$, so 
Condition (ii) of Theorem 4 holds. Condition (iii) of Theorem 4 is trivially satisfied by $\left\{g^{1}\right\}$.

We conclude this section by observing that farsightedly stable sets may depend in a non-monotonic way on the degree of farsightedness by showing that $\left\{g^{1}\right\}$ is not a level-3 farsightedly stable set in Example 4 , but is a level- $K$ farsightedly stable set for every $K \geq n^{\prime}+1$.

Example 5. Consider the same network situation as in Example 4, so $\left\{g^{1}\right\}$ is a level-2 farsightedly stable set. We argue by contradiction that it is not a level-3 farsightedly stable set.

Suppose that $\left\{g^{1}\right\}$ is a level-3 farsightedly stable set. Consider a deviation by Player 1, who cuts the link with Player 2 to arrive at the network $g^{0}$. When Player 1 has only two degrees of farsightedness, he might fear a further move to $g^{3}$, which would deter the deviation. With three degrees of farsightedness, Player 1 realizes that the threat of ending in $g^{3}$ is not credible, since $g^{3}$ does not belong to the level3 farsightedly stable set $\left\{g^{1}\right\}$. Since the set $f_{2}\left(g^{0}\right) \backslash f_{1}\left(g^{0}\right)=\emptyset$, the deviation by Player 1 to $g^{0}$ is not deterred according to Definition 3.

However, when players are sufficiently farsighted, $\left\{g^{1}\right\}$ reemerges as a level- $K$ farsightedly stable set. We consider some $K \geq n^{\prime}+1$ and verify that $\left\{g^{1}\right\}$ satisfies the conditions of Theorem 5. As before, the only deviations from $g^{1}$ are to $g^{0}, g^{4}$, and $g^{5}$. Since $f_{n^{\prime}-1}\left(g^{0}\right), f_{n^{\prime}-1}\left(g^{4}\right)$, and $f_{n^{\prime}-1}\left(g^{5}\right)$ all contain $g^{1}$, such deviations are deterred, and Condition (i) of Theorem 3 is satisfied. Since $g^{1} \in f_{n^{\prime}-1}\left(g^{\prime}\right) \subseteq f_{n^{\prime}-1}^{\infty}\left(g^{\prime}\right)$ for all $g^{\prime} \in \mathbb{G} \backslash\left\{g^{1}\right\}$, we know that Condition (ii) of Theorem 3 is satisfied by $\left\{g^{1}\right\}$. Condition (iii) of Theorem 4 is trivially satisfied. It follows that $\left\{g^{1}\right\}$ is a level- $K$ farsightedly stable set for every $K \geq n^{\prime}+1$.

\section{Sufficient Conditions for Level- $K$ Farsighted Sta- bility}

In this section, we present two sets of sufficient conditions for a set to be level- $K$ farsightedly stable. In many examples, these conditions are easy to verify.

A refinement of pairwise stability is obtained when we require the network $g$ to defeat every other adjacent network, so $g \in f_{1}\left(g^{\prime}\right)$ for every network $g^{\prime}$ adjacent to 
$g$. We call such a network $g$ pairwise dominant. The following definition generalizes this idea and allows for farsighted improving paths of any length $K$.

Definition 4. For $K \geq 1$, a network $g$ is level- $K$ pairwise dominant if for every $g^{\prime}$ adjacent to $g$ it holds that $g \in f_{K}\left(g^{\prime}\right)$. The set of level-K pairwise dominant networks is denoted by $D_{K}$.

It follows immediately from the definition that $D_{1} \subseteq P_{1}$. For generic allocation rules, the concepts of pairwise stability and pairwise dominance coincide, $D_{1}=P_{1}$. This coincidence does not hold for values of $K$ greater than or equal to 2. By Lemma 1 it follows that $D_{K} \subseteq D_{K+1}$, whereas $P_{K} \supseteq P_{K+1}$.

The first set of sufficient conditions applies to the case where $K=1$.

Theorem 8. If $g \in P_{1}$ and for every $g^{\prime} \in \mathbb{G} \backslash\{g\}$ it holds that $g \in f_{1}^{\infty}\left(g^{\prime}\right)$, then $\{g\}$ is the unique level-1 farsightedly stable set.

Proof. We show that $\{g\}$ is a level-1 farsightedly stable set by applying Theorem 2 . The uniqueness then follows from Theorem 3. Since $g \in P_{1}$ it holds that $f_{1}(g)=\{g\}$, so for a deviation from $g$ to $g^{\prime}=g+i j$ it holds that $\left(Y_{i}\left(g^{\prime}\right), Y_{j}\left(g^{\prime}\right)\right)=\left(Y_{i}(g), Y_{j}(g)\right)$ or $Y_{i}\left(g^{\prime}\right)<Y_{i}(g)$ or $Y_{j}\left(g^{\prime}\right)<Y_{j}(g)$ and for a deviation from $g$ to $g^{\prime}=g-i j$ it holds that $Y_{i}\left(g^{\prime}\right) \leq Y_{i}(g)$ and $Y_{j}\left(g^{\prime}\right) \leq Y_{j}(g)$, so Condition (i) of Theorem 2 is satisfied. Conditions (ii) and (iii) of Theorem 2 are trivially satisfied.

The next result applies when $K \geq 2$.

Theorem 9. Consider some $K \geq 2$. If $g \in D_{J}$ for some $J<K$ and for every $g^{\prime} \in \mathbb{G} \backslash\{g\}$ it holds that $g \in f_{K}^{\infty}\left(g^{\prime}\right)$, then $\{g\}$ is a level-K farsightedly stable set. If, moreover, $g \in P_{K}$, then $\{g\}$ is the unique level-K farsightedly stable set.

Proof. We start by showing that $\{g\}$ is a level- $K$ farsightedly stable set.

We first consider $K=2$ and apply Theorem 4 . If $K=2$, then the only possibility is that $J=1$, so $g \in D_{1}$, or equivalently $g \in \tilde{f}_{1}(\bar{g})$ for every $\bar{g}$ adjacent to $g$. Condition (i) of Theorem 4 is satisfied since a deviation from $g$ to $\bar{g}$ is deterred by the return to $g \in \tilde{f}_{1}(\bar{g})$. Conditions (ii) and (iii) of Theorem 4 are trivially satisfied.

We next consider $K \geq 3$ and apply Definition 3. Since $g \in D_{J}$ for some $J<K$, it holds that $g \in f_{J}(\bar{g}) \subseteq f_{K-1}(\bar{g})$ for every $\bar{g}$ adjacent to $g$, where the inclusion uses Lemma 1. It holds that either $g \in f_{K-2}(\bar{g})$, so $g \in f_{K-2}(\bar{g}) \cap\{g\}$, or $g \notin f_{K-2}(\bar{g})$, so $g \in f_{K-1}(\bar{g}) \backslash f_{K-2}(\bar{g})$. Condition (i) of Definition 3 is satisfied since a deviation 
from $g$ to $\bar{g}$ is deterred by the return to $g \in\left[f_{K-2}(\bar{g}) \cap\{g\}\right] \cup\left[f_{K-1}(\bar{g}) \backslash f_{K-2}(\bar{g})\right]$. Conditions (ii) and (iii) of Definition 3 are trivially satisfied.

We complete the proof by showing that $\{g\}$ is the unique level- $K$ farsightedly stable set if in addition $g \in P_{K}$. Since $g \in f_{K}^{\infty}\left(g^{\prime}\right)$ for every $g^{\prime} \in \mathbb{G} \backslash\{g\}$ and $g \in P_{K}$, we have that $P_{K}=\{g\}$, and therefore $P_{K}$ is a level- $K$ farsightedly stable set. Corollary 1 yields the desired result.

The conditions of Theorems 8 and 9 are usually easy to verify. To show that $g \in P_{1}$ requires that $f_{1}(g)$ does not contain networks different from $g$. To show that $g \in f_{1}^{\infty}\left(g^{\prime}\right)$ for all $g^{\prime} \neq g$, we have to find a sequence of farsighted improving paths of length one that connect $g^{\prime}$ to $g$. In Theorem 9 the requirement of Theorem 8 that $g$ belongs to $P_{1}$ is replaced by the requirement that $g \in D_{J}$ for some $J<K$, so we have to show that $g \in f_{J}\left(g^{\prime}\right)$ for all $g^{\prime}$ adjacent to $g$. The higher $J$, the weaker is this requirement, so we could replace the requirement $g \in D_{J}$ for some $J<K$ by $g \in D_{K-1}$. To show that $g \in f_{K}^{\infty}\left(g^{\prime}\right)$ for all $g^{\prime} \neq g$, we have to find a sequence of farsighted improving paths of length at most $K$ that connect $g^{\prime}$ to $g$. Very often the analysis of farsighted improving paths of small lengths is already sufficient. The higher $K$, the easier it is to satisfy the conditions of Theorem 9 and to find a singleton level- $K$ farsightedly stable set. Finally, to show that $g \in P_{K}$ requires that $f_{K}(g)$ does not contain networks different from $g$. This requirement is more difficult to satisfy for increasing values of $K$.

We show next how Theorems 8 and 9 can be used to analyze the stability of $g^{3}$ in Example 4. In Example 4 it holds that $g^{3} \in P_{1}$ and for every $g \in \mathbb{G} \backslash\left\{g^{3}\right\}$, $g^{3} \in f_{1}^{\infty}(g)$. We can then apply Theorem 8 to conclude that $\left\{g^{3}\right\}$ is the unique level-1 farsightedly stable set.

In Example 4 it also holds that $g^{3} \in D_{1}$. Since for every $g \in \mathbb{G} \backslash\left\{g^{3}\right\}, g^{3} \in f_{1}^{\infty}(g)$, we have by Lemma 2 that $g^{3} \in f_{K}^{\infty}(g)$ for every $K \geq 2$. We can then apply Theorem 9 to conclude that $\left\{g^{3}\right\}$ is a level- $K$ farsightedly stable set for any value of $K \geq 2$.

We have illustrated in Example 4 that there are other farsightedly stable sets for higher values of $K$, in particular $\left\{g^{1}\right\}$ can be sustained as a farsightedly stable set for higher values of $K$. Indeed, for $K \geq 2, f_{K}\left(g^{3}\right)$ contains networks different from $g^{3}$, so the condition $g^{3} \in P_{K}$ in Theorem 9, which is sufficient for uniqueness of $\left\{g^{3}\right\}$ as a level- $K$ farsightedly stable set, does not hold.

In Example 4, $\left\{g^{1}\right\}$ has been shown to be a level-2 farsightedly stable set. In Example 5 we have argued that $g^{1}$ is not a level-3 farsightedly stable set. We show 
next that Theorem 9 can be used to show that $\left\{g^{1}\right\}$ is a level- $K$ farsightedly stable set for any $K \geq 4$. The adjacent networks of $g^{1}$ are $g^{0}, g^{4}$, and $g^{5}$. It follows from Table 1 that $f_{3}\left(g^{0}\right), f_{3}\left(g^{4}\right)$, and $f_{3}\left(g^{5}\right)$ all contain $g^{1}$, so $g^{1} \in D_{3}$. We have already argued in Example 4 that for every $g^{\prime} \in \mathbb{G} \backslash\left\{g^{1}\right\}$ it holds that $g^{1} \in f_{2}^{\infty}\left(g^{\prime}\right)$ so, by Lemma 2, we have that $g^{1} \in f_{K}^{\infty}\left(g^{\prime}\right)$ for all $K \geq 2$. Combining the conclusions in the previous two sentences and applying Theorem 9 proves that $\left\{g^{1}\right\}$ is a level- $K$ farsightedly stable set for any $K \geq 4$.

\section{$7 \quad$ Efficiency and Stability}

We now turn to the question of how level- $K$ farsighted stability is related to the efficiency of networks. A network $g$ is strongly efficient if $\sum_{i \in N} Y_{i}(g)>\sum_{i \in N} Y_{i}\left(g^{\prime}\right)$ for all $g^{\prime} \neq g$. Assume that there is a network $\widetilde{g}$ that strictly Pareto dominates all other networks. That is, $Y_{i}(\widetilde{g})>Y_{i}(g)$ for all $i \in N$ and for all $g \in \mathbb{G} \backslash\{\widetilde{g}\}$. Hence, $\widetilde{g}$ is both Pareto efficient and strongly efficient.

Theorem 10. Assume that the network $\widetilde{g}$ strictly Pareto dominates all networks $g \in \mathbb{G} \backslash\{\widetilde{g}\}$. Then, $\{\widetilde{g}\}$ is the unique level-K farsightedly stable set for all $K \geq$ $n(n-1) / 2$.

Proof. First, we show that $\widetilde{g} \in D_{1}$. For all $g \in \mathbb{G} \backslash\{\widetilde{g}\}$, it holds that $Y_{i}(\widetilde{g})>Y_{i}(g)$ for all $i \in N$, so we have that $\widetilde{g} \in f_{1}(g)$ for any network $g$ adjacent to $\widetilde{g}$, so $\widetilde{g} \in D_{1}$. Moreover, for every $g \in \mathbb{G} \backslash\{\widetilde{g}\}$, we have that $\widetilde{g} \in f_{K}(g)$ when $K \geq n(n-1) / 2$. Indeed, all players like to move from $g$ to $\widetilde{g}$ given that $Y_{i}(\widetilde{g})>Y_{i}(g)$ for all $i \in N$, and the maximum number of links that one needs to cut or form from $g$ in order to reach $\widetilde{g}$ is equal to the number of links in the complete network, $n(n-1) / 2$. It follows that $\widetilde{g} \in f_{K}^{\infty}(g)$ for all $g \in \mathbb{G} \backslash\{\widetilde{g}\}$. Finally, since $\widetilde{g}$ strictly Pareto dominates all other networks, we have that $f_{K}(\widetilde{g})=\{\widetilde{g}\}$ for all $K \geq 1$. Thus, $\widetilde{g} \in P_{K}$ for all $K \geq 1$, and by Theorem 9 we have that $\{\widetilde{g}\}$ is the unique level- $K$ farsightedly stable set for all $K \geq n(n-1) / 2$.

In the investment game of Example 1, the complete network $g^{N}$ strictly Pareto dominates all other networks. Hence, from Theorem 10 we have that $\left\{g^{N}\right\}$ is the unique level- $K$ farsightedly stable set for all $K \geq n(n-1) / 2$. Observe that Theorem 10 holds for levels of farsightedness relatively small as compared to the total number of possible networks. 
There are many situations where a Pareto dominating network does not exist. We therefore turn our attention to allocation rules satisfying increasing returns to link creation. An allocation rule $Y$ displays no externalities across components (NEC) if for every $g \in \mathbb{G}$, for every $h \in C(g)$, we have $Y_{i}(g)=Y_{i}(h)$ for all $i \in N(h)$ and $Y_{i}(g)=0$ for all $i \in N \backslash N(g)$. In particular, it holds that $Y_{i}\left(g^{\emptyset}\right)=0$ for all $i \in N$. If an allocation rule satisfies NEC, then it is sufficient to specify the allocation rule for connected networks. The set of connected networks is denoted by $\mathbb{C}$.

Consider some allocation rule $Y$ and let

$$
C^{+}(Y)=\left\{h \in \mathbb{C} \backslash\left\{g^{\emptyset}\right\} \mid \sum_{i \in N} Y_{i}(h) \geq 0\right\}
$$

be the set of non-empty connected networks with non-negative aggregate payoffs. The allocation rule $Y$ satisfies increasing returns to link creation (IRL) ${ }^{14}$ if:

(i) $Y$ satisfies NEC.

(ii) If $h \in C^{+}(Y)$ and $h \subseteq h^{\prime} \in \mathbb{C}$, then $h^{\prime} \in C^{+}(Y)$.

(iii) If $h \in C^{+}(Y)$ and $i j \in h$, then $Y_{i}(h-i j) \leq Y_{i}(h)$ and $Y_{j}(h-i j) \leq Y_{j}(h)$ with at least one inequality holding strictly.

(iv) There exists $h^{\prime} \in C^{+}(Y)$ such that for all $h \in \mathbb{C}$ with $h \varsubsetneqq h^{\prime}$, for all $i \in N\left(h^{\prime}\right)$, we have $Y_{i}(h)<Y_{i}\left(h^{\prime}\right)$.

If an allocation rule satisfies increasing returns to link creation, then by Condition (iv) there is a non-empty connected network $h^{\prime}$ for which the payoff of all players having at least one link is greater than the payoffs they could obtain in any network $h \varsubsetneqq h^{\prime}$. If we take $h=g^{\emptyset}$, then it follows that $Y_{i}\left(h^{\prime}\right)>0$ for all $i \in N\left(h^{\prime}\right)$. By Condition (ii), the aggregate payoffs in any connected network containing $h^{\prime}$ are non-negative, and it follows in particular that $\sum_{i \in N} Y_{i}\left(g^{N}\right) \geq 0 .{ }^{15}$ Notice that in

\footnotetext{
${ }^{14}$ Dutta, Ghosal and Ray (2005) defines the property of increasing returns to link creation for a value function. A value function satisfies this property if there is a threshold network for which the value is non-negative, and each time a new link is added to this threshold network, both aggregate payoffs and payoffs of players who are adding a link to the network increase. Here, we translate the main idea behind this property to an allocation rule.

${ }^{15}$ Dutta, Ghosal and Ray (2005) defines the property of increasing returns to link creation for a value function by imposing the value of each network $h \supsetneq h^{\prime}$ to be greater than the value of $h^{\prime}$ and, in particular, that the complete network generates the highest possible value, i.e. the complete network is the strongly efficient network.
} 
connected networks containing $h^{\prime}$, some players may have negative payoffs, but that their payoffs increase when making additional links by Condition (iii). The investment game in Example 1 satisfies IRL as well as generalizations of the investment game where payoffs to players are positive as soon as the network has more links than some threshold value. Another model that satisfies IRL is the symmetric connections model of Jackson and Wolinsky (1996) when the cost for maintaining a link is small, $c<\delta(1-\delta)$.

We now show that if the allocation rule satisfies IRL, then there exists a value of $K^{\prime}$ such that, for all $K \geq K^{\prime},\left\{g^{N}\right\}$ is a level- $K$ farsightedly stable set. A minimal network $h^{\prime}$ as in Condition (iv) of IRL is called critical. Let $\widetilde{K}=\min \left\{\# h^{\prime} \mid h^{\prime}\right.$ is a critical network $\}$ be the number of links in the critical network with the lowest number of links, and let $\bar{K}$ be the highest number of links in a connected network that does not contain any critical network. Then $K^{\prime}$ can be taken equal to $\max \{\widetilde{K}, \bar{K}\}$. For the investment game in Example 1 it holds that $\widetilde{K}=n(n-1) / 2$ and $\bar{K}=$ $n(n-1) / 2-1$, so $K^{\prime}=n(n-1) / 2$.

Theorem 11. Let the allocation rule $Y$ satisfy IRL. Then $\left\{g^{N}\right\}$ is a level-K farsightedly stable set for all $K \geq \max \{\widetilde{K}, \bar{K}\}$.

Proof. First, we show that $g^{N} \in D_{1}$. We argued before that IRL implies $g^{N} \in$ $C^{+}(Y)$. By Condition (iii) of IRL, we have that $Y_{i}\left(g^{N}\right) \geq Y_{i}\left(g^{N}-i j\right)$ and $Y_{j}\left(g^{N}\right) \geq$ $Y_{j}\left(g^{N}-i j\right)$ with at least one inequality holding strictly. It follows that $g^{N} \in f_{1}(g)$ for any network $g$ adjacent to $g^{N}$, so $g^{N} \in D_{1}$.

To apply Theorem 9 , or Theorem 8 when $K=1$, we need to show that $g^{N} \in$ $f_{K}^{\infty}(g)$ for every $g \neq g^{N}$.

(a) First, consider any network $g^{\prime} \in \mathbb{G}$ having a component $h^{\prime} \in C\left(g^{\prime}\right)$ such that $\sum_{i \in N} Y_{i}\left(h^{\prime}\right)<0$. By Condition (ii) of IRL it follows for any non-empty and connected $h \subseteq h^{\prime}$ that $\sum_{i \in N} Y_{i}(h)<0$, so $h^{\prime}$ does not contain a critical network. Moreover, for any non-empty $h \subseteq h^{\prime}$ there is a player $i \in N(h)$ who has a payoff $Y_{i}(h)<0$, and so $i$ has incentives to cut a link, say link $i j$, foreseeing the empty network where $Y_{i}\left(g^{\emptyset}\right)=0$. We have that $g^{\emptyset} \in f_{\bar{K}}\left(h^{\prime}\right)$, and therefore that $g^{\prime} \backslash h^{\prime} \in$ $f_{\bar{K}}\left(g^{\prime}\right)$.

If $g^{\prime} \backslash h^{\prime}$ has a component $h^{\prime \prime} \in C\left(g^{\prime} \backslash h^{\prime}\right)$ such that $\sum_{i \in N} Y_{i}\left(h^{\prime \prime}\right)<0$, then we can use the argument of the previous paragraph to obtain that $g^{\prime} \backslash\left(h^{\prime} \cup h^{\prime \prime}\right) \in f_{\bar{K}}\left(g^{\prime} \backslash h^{\prime}\right)$, and therefore that $g^{\prime} \backslash\left(h^{\prime} \cup h^{\prime \prime}\right) \in f_{\bar{K}}^{2}\left(g^{\prime}\right)$. Repeating this argument a finite number 
of times, we arrive at a network $g$ that is either equal to $g^{\emptyset}$ or has only components belonging to $C^{+}(Y)$.

(b) Second, consider the empty network $g^{\emptyset}$. From Condition (iv) of IRL, there is a network $h^{\prime} \in C^{+}(Y)$ such that for all $h \in \mathbb{C}$ with $h \subsetneq h^{\prime}$ it holds that $Y_{i}\left(h^{\prime}\right)>Y_{i}(h)$ for all $i \in N\left(h^{\prime}\right)$. In particular, we have that $Y_{i}\left(h^{\prime}\right)>Y_{i}\left(g^{\emptyset}\right)$ for all $i \in N\left(h^{\prime}\right)$. Let $h^{\prime}$ be such a network with $\widetilde{K}$ links. The players in $N\left(h^{\prime}\right)$ have incentives to form sequentially the missing links in $g^{\emptyset}$ foreseeing $h^{\prime}$, so $h^{\prime} \in f_{\widetilde{K}}\left(g^{\emptyset}\right)$.

(c) Third, consider any non-empty network $g^{\prime} \neq g^{N}$ having only components in $C^{+}(Y)$ and let $i$ be a player in $N\left(g^{\prime}\right)$ with degree $d_{i}\left(g^{\prime}\right)<n-1$. Let $j \in N$ be such that ij $\notin g^{\prime}$. It follows from Conditions (i) and (ii) of IRL that all components of $g^{\prime}+i j$ belong to $C^{+}(Y)$. We find by Conditions (i) and (iii) of IRL that $Y_{i}\left(g^{\prime}+i j\right) \geq$ $Y_{i}\left(g^{\prime}\right)$ and $Y_{j}\left(g^{\prime}+i j\right) \geq Y_{j}\left(g^{\prime}\right)$ with at least one inequality strict, so $g^{\prime}+i j \in f_{1}\left(g^{\prime}\right)$. Repeating this argument $m$ times, where $m$ is the number of links in $g^{N} \backslash g^{\prime}$, we find that $g^{N} \in f_{1}^{m}\left(g^{\prime}\right)$.

Theorem 9 now implies that $\left\{g^{N}\right\}$ is a level- $K$ farsightedly stable set for all $K \geq \max \{\widetilde{K}, \bar{K}\}$.

In the symmetric connections model, there is a sequence of improving paths of length 1 from any $g \neq g^{N}$ to $g^{N}$ when $c<\delta(1-\delta)$. Hence, if $c<\delta(1-\delta)$, then $g^{N} \in f_{1}^{\infty}(g)$ for any $g \neq g^{N}$, and $\left\{g^{N}\right\}$ is the unique level- $K$ farsightedly stable set for all $K \geq 1$. In this model, the complete network is strongly efficient.

\section{Criminal Networks}

There is empirical evidence suggesting that peer effects and the structure of social interactions matter strongly in explaining an individual's own criminal or delinquent behavior. ${ }^{16}$ Calvo-Armengol and Zenou (2004) provide a network analysis of criminal behavior. They develop a model where criminals compete with each other in criminal activities but benefit from being friends with other criminals by improving their knowledge of the crime business. Individuals decide first whether to work or to become a criminal and then they choose the crime effort to exert if criminals. ${ }^{17}$

\footnotetext{
${ }^{16}$ See Patacchini and Zenou (2008) among others.

${ }^{17}$ Calvo-Armengol and Zenou (2004) mostly focus on the case where the network is exogenously given. They show that multiple equilibria with different members of active criminals and levels of involvement in crime business may coexist.
} 
Here, we present a simplified version of their model, which puts emphasis on the formation of links and keeps the level of criminal activities of the players fixed.

Throughout this section, we assume $n \geq 3$. The players are referred to as criminals. Given some criminal network $g$, the elements of $P(g)$ are called criminal groups. Each criminal group $S$ has a positive probability $p_{S}(g)$ of winning the loot $B>0$. It is assumed that the bigger the criminal group, the higher its probability of getting the loot. This assumption captures the idea that delinquents learn from other criminals belonging to the same group how to commit crime in a more efficient way by sharing the know-how about the technology of crime. We assume that the probability of winning the loot is given by $p_{S}(g)=\# S / n$.

The network architecture determines how the loot is shared among the criminals in the group. Consider some Player $i \in N$ and let $S \in P(g)$ be the criminal group $i$ belongs to. We define $c_{i}(g)=\max _{j \in S} d_{j}(g)$ as the maximum degree in this criminal group. A criminal $i$ who is part of a group $S \in P(g)$ expects a share $\alpha_{i}(g)$ of the loot given by

$$
\alpha_{i}(g)= \begin{cases}\frac{1}{\#\left\{j \in S \mid d_{j}(g)=c_{j}(g)\right\}}, & \text { if } d_{i}(g)=c_{i}(g) \\ 0, & \text { otherwise }\end{cases}
$$

That is, within each criminal group, the criminal that has the highest number of links gets the loot. If two or more criminals have the highest number of links, then they share the loot equally among them.

Criminal $i$ has a probability $q_{i}(g)$ of being caught, in which case his rewards are punished at a rate $\phi>0$. It is assumed that the higher the number of links a criminal has, the lower his individual probability of being caught. We assume that the probability of being caught is simply given by

$$
q_{i}(g)=\frac{n-1-d_{i}(g)}{n} .
$$

The total payoffs of criminal $i$ belonging to criminal group $S \in P(g)$ are therefore equal to

$$
\begin{aligned}
Y_{i}(g) & =p_{S}(g) \alpha_{i}(g)\left(1-q_{i}(g) \phi\right) B \\
& = \begin{cases}\frac{\# S}{n} \frac{1}{\#\left\{j \in S \mid d_{j}(g)=c_{i}(g)\right\}}\left(1-\frac{n-1-d_{i}(g)}{n} \phi\right) B, & \text { if } d_{i}(g)=c_{i}(g), \\
0, & \text { otherwise. }\end{cases}
\end{aligned}
$$

We require $\phi<n /(n-1)$ to guarantee that payoffs are non-negative and positive for a player with the highest degree in his group. 


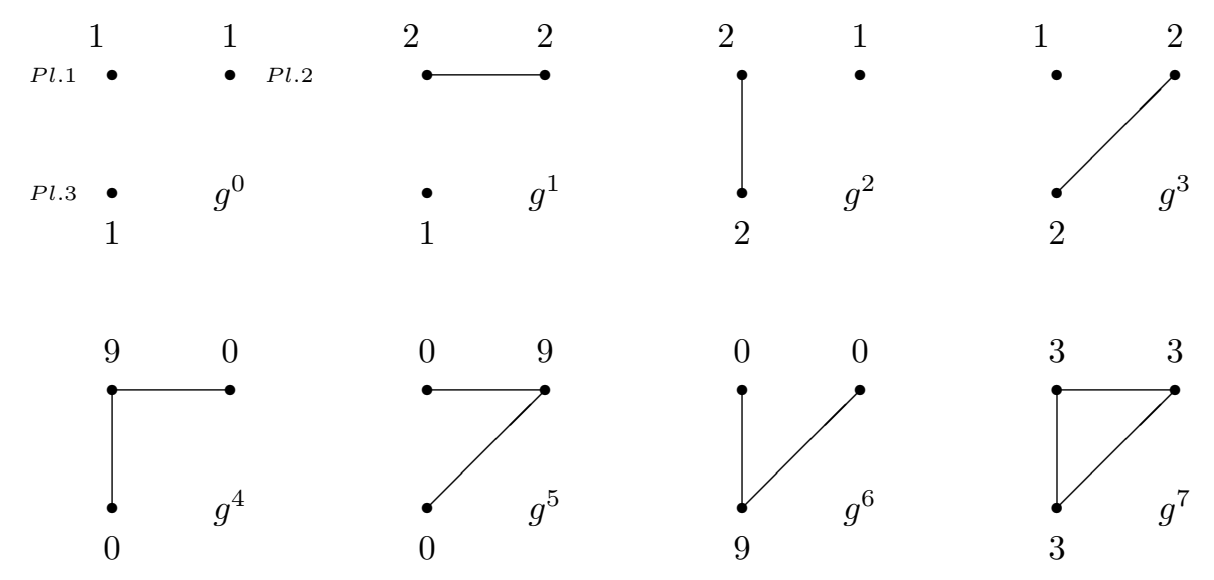

Figure 3: The 3-player criminal networks.

Figure 3 presents the payoffs for 3 -player criminal networks with $B=9$ and $\phi=1$. Table 2 shows the farsighted improving paths for the different possible values of $K$. It can be verified that the farsighted improving paths for the 3-player case do not depend on the specific choices for $B$ and $\phi$.

\begin{tabular}{|c||c|c|c|}
\hline$g$ & $\tilde{f}_{1}(g)$ & $\tilde{f}_{2}(g)$ & $\tilde{f}_{K}(g), K \geq 3$ \\
\hline \hline$g^{0}$ & $g^{1}, g^{2}, g^{3}$ & $g^{1}, g^{2}, g^{3}$ & $g^{1}, g^{2}, g^{3}, g^{7}$ \\
\hline$g^{1}, g^{2}, g^{3}$ & & $g^{7}$ & $g^{7}$ \\
\hline$g^{4}$ & $g^{1}, g^{2}, g^{7}$ & $g^{1}, g^{2}, g^{7}$ & $g^{1}, g^{2}, g^{3}, g^{7}$ \\
\hline$g^{5}$ & $g^{1}, g^{3}, g^{7}$ & $g^{1}, g^{3}, g^{7}$ & $g^{1}, g^{2}, g^{3}, g^{7}$ \\
\hline$g^{6}$ & $g^{2}, g^{3}, g^{7}$ & $g^{2}, g^{3}, g^{7}$ & $g^{1}, g^{2}, g^{3}, g^{7}$ \\
\hline$g^{7}$ & & & \\
\hline
\end{tabular}

Table 2: The elements of $\tilde{f}_{K}(g)$ for 3 -player criminal networks with $B=9$ and $\phi=1$

For the three-player case, we compute the closed cycles and use Theorem 3 to conclude that $G_{1}=P_{1}=\left\{g^{1}, g^{2}, g^{3}, g^{7}\right\}$ is the level-1 farsightedly stable set, so $G_{1}$ consists of all pairwise stable networks. There are many networks that are stable when players are myopic.

For $K \geq 2$, we apply Theorem 9 to show that $G_{K}=\left\{g^{7}\right\}$ is the unique level$K$ farsightedly stable set. It holds that $g^{7} \in D_{1}$ and $g^{7} \in f_{2}^{\infty}(g)$ for every $g \neq$ $g^{7}$, so $\left\{g^{7}\right\}$ is a level- $K$ farsightedly stable set. Since $g^{7} \in P_{K}$, it follows from 
Theorem 9 that $\left\{g^{7}\right\}$ is the unique level- $K$ farsightedly stable set. If criminals behave myopically, they may not go beyond forming a single link in the three player case. But with a degree of farsightedness of at least 2 , the complete criminal network emerges as the unique prediction.

The remainder of this section is devoted to the analysis of criminal networks with a general number $n$ of players. As in the 3-criminal case, there are many networks that are pairwise stable in the $n$-person case. The complete network is easily verified to be pairwise stable. The generalization of the networks $g^{1}, g^{2}$, and $g^{3}$ for the 3 -criminal case to the $n$-criminal case would be any network consisting of complete components, where no two components have the same degree. But also any network with a single component where all players have a degree at least equal to two and one player has a degree that is at least two times higher than the degree of any other player is pairwise stable.

We will argue next that $\left\{g^{N}\right\}$ is a level- $K$ farsightedly stable set whenever $K \geq$ $n-1$.

We show first that the complete network is pairwise dominant.

Lemma 3. For criminal networks it holds that $g^{N} \in D_{1}$.

Proof. Consider the network $g^{N}-i j$ for some $i j$. It holds that

$$
d_{i}\left(g^{N}-i j\right)=d_{j}\left(g^{N}-i j\right)<c_{i}\left(g^{N}-i j\right)=c_{j}\left(g^{N}-i j\right)
$$

So

$$
Y_{i}\left(g^{N}-i j\right)=Y_{j}\left(g^{N}-i j\right)=0<Y_{i}\left(g^{N}\right)=Y_{j}\left(g^{N}\right)
$$

and $g^{N} \in f_{1}\left(g^{N}-i j\right)$. We have shown that $g^{N} \in D_{1}$.

We show next that the complete network can be reached from any starting network by repeated application of at most $n-1$ degrees of farsightedness.

Lemma 4. For criminal networks, it holds for every $g \in \mathbb{G} \backslash\left\{g^{N}\right\}$ that $g^{N} \in f_{n-1}^{\infty}(g)$. Proof.

Step 1. If $g$ has a component which is not complete, then there is $g^{\prime} \in f_{n-1}(g)$ such that $g \subsetneq g^{\prime}$.

Let $S \in P(g)$ be a criminal group such that some internal links are missing, $g_{\mid S} \neq g^{S}$. 
If for every $i \in S$ it holds that $d_{i}(g)=c_{i}(g)$, so all players in $S$ have the same degree, then any two players $i$ and $j$ in $S$ create a link to form the network $g+i j$ and improve their payoffs since the increase in their degree increases the share in the loot and lowers the probability of being caught for both players, $\alpha_{i}(g+i j)>\alpha_{i}(g)$, $\alpha_{j}(g+i j)>\alpha_{j}(g), q_{i}(g+i j)<q_{i}(g)$, and $q_{j}(g+i j)<q_{j}(g)$, so $Y_{i}(g+i j)>Y_{i}(g)$ and $Y_{j}(g+i j)>Y_{j}(g)$. We have that $g \rightarrow_{1} g+i j$, so clearly $g+i j \in f_{n-1}(g)$.

If the players in $S$ do not all have the same degree, let $i \in S$ be a player with $d_{i}(g)<c_{i}(g)$, so $\alpha_{i}(g)=0$ and therefore $Y_{i}(g)=0$.

If $c_{i}(g)=\# S-1$, then Player $i$ consecutively links to all players $j \in S$ such that ij $\notin g$, thereby forming a network $g^{\prime}$ where he has degree $\# S-1$. The payoffs of Player $i$ are in every step equal to $Y_{i}(g)=0$ until the final step, where his payoffs increase to $Y_{i}\left(g^{\prime}\right)>0$. Every player $j$ that $i$ links to has degree below \#S-1 and therefore payoffs equal to $0 \leq Y_{j}\left(g^{\prime}\right)$. We have that $g^{\prime} \in f_{\# S-2}(g) \subseteq f_{n-1}(g)$.

If $c_{i}(g)<\# S-1$, then let $j \in S$ be a player with $d_{j}(g)=c_{i}(g)$. Player $i$ (with $d_{i}(g)<c_{i}(g)$ ) links with Player $j$ to form the network $g+i j$. It holds that $Y_{i}(g+i j)=Y_{i}(g)=0$ and $Y_{j}(g+i j)>Y_{j}(g)>0$, since $\alpha_{j}(g+i j) \geq \alpha_{j}(g)$ and $q_{j}(g+i j)<q_{j}(g)$. In this case we have that $g \rightarrow_{1} g+i j$, so clearly $g+i j \in f_{n-1}(g)$.

Step 2. If all components of $g$ are complete and $g \neq g^{N}$, then there is $g^{\prime} \in f_{n-1}(g)$ such that $g \subsetneq g^{\prime}$.

The assumptions of Step 2 imply that $g$ consists of at least two criminal groups. Let $S^{1}$ and $S^{2}$ be two criminal groups in $P(g)$.

If $\# S^{1}=\# S^{2}$, then form a link between a Player $i \in S^{1}$ and a Player $j \in S^{2}$. Since $q_{i}(g)>q_{i}(g+i j)$, we have that

$$
Y_{i}(g)=\frac{1}{n}\left(1-q_{i}(g) \phi\right) B<\frac{\# S_{1}}{n}\left(1-q_{i}(g+i j) \phi\right) B=Y_{i}(g+i j) .
$$

By the same calculation, it follows that $Y_{j}(g)<Y_{j}(g+i j)$, so $g \rightarrow_{1} g+i j$, and therefore $g+i j \in f_{n-1}(g)$.

Otherwise, it holds without loss of generality that $\# S^{1}<\# S^{2}$. Select some player $i \in S^{1}$ and a set $J$ consisting of $\# S^{2}+1-\# S^{1}$ players in $S^{2}$, who link consecutively to Player $i$ to form network $g^{\prime}$. The resulting finite sequence of networks is denoted $g_{0}, \ldots, g_{K}$ with $g_{0}=g$ and $g_{K}=g^{\prime}$. Notice that $K \leq n-1$. We show next that for every $k \in\{0, \ldots, K-1\},\left(Y_{i}\left(g_{k}\right), Y_{j_{k}}\left(g_{k}\right)\right)<\left(Y_{i}\left(g_{K}\right), Y_{j_{k}}\left(g_{K}\right)\right)$, where $j_{k} \in J$ is such that $g_{k+1}=g_{k}+i j_{k}$, thereby proving that $\left(g_{0}, \ldots, g_{K}\right)$ is a farsighted improving path and completing the proof of Step 2. 
For every player $j \in J$ we have

$$
d_{j}\left(g_{K}\right)=d_{i}\left(g_{K}\right)=c_{i}\left(g_{K}\right)
$$

and for all other players the degree is strictly less than $c_{i}\left(g_{K}\right)$, so

$$
Y_{j}\left(g_{K}\right)=Y_{i}\left(g_{K}\right)=\frac{\# S^{1}+\# S^{2}}{n} \frac{1}{\# S^{2}+2-\# S^{1}}\left(1-q_{i}\left(g_{K}\right) \phi\right) B .
$$

For $k=0$, we have

$$
\begin{aligned}
Y_{i}\left(g_{0}\right) & =\frac{1}{n}\left(1-q_{i}(g) \phi\right) B<Y_{i}\left(g_{K}\right), \\
Y_{j_{0}}\left(g_{0}\right) & =\frac{1}{n}\left(1-q_{j_{0}}(g) \phi\right) B<Y_{j_{0}}\left(g_{K}\right),
\end{aligned}
$$

where we use $q_{i}\left(g_{0}\right)>q_{i}\left(g_{K}\right)$ and $q_{j_{0}}\left(g_{0}\right)>q_{j_{0}}\left(g_{K}\right)$ to get the strict inequalities.

For $k=1, \ldots, K-1$, it holds that Player $i$ is connected to Player $j_{0}$, so $d_{i}\left(g_{k}\right)<$ $d_{j_{0}}\left(g_{k}\right)=c_{i}\left(g_{k}\right)$, so $\alpha_{i}\left(g_{k}\right)=0$ and $0=Y_{i}\left(g_{k}\right)<Y_{i}\left(g_{K}\right)$. Similarly, it holds that Player $j_{k}$ is connected to Player $j_{0}$, so $d_{j_{k}}\left(g_{k}\right)<d_{j_{0}}\left(g_{k}\right)=c_{j_{k}}\left(g_{k}\right)$, so $\alpha_{j_{k}}\left(g_{k}\right)=0$ and $0=Y_{j_{k}}\left(g_{k}\right)<Y_{j_{k}}\left(g_{K}\right)$.

Step 3. For every $g \in \mathbb{G} \backslash\left\{g^{N}\right\}$, it holds that $g^{N} \in f_{n-1}^{\infty}(g)$.

By combining the results of Step 1 and Step 2, we have that for every $g \in \mathbb{G} \backslash\left\{g^{N}\right\}$, there is $g^{\prime} \in f_{n-1}(g)$ with strictly more links than $g$. Since the complete network $g^{N}$ has $n(n-1) / 2$ links, we find that $g^{N} \in f_{n-1}^{n(n-1) / 2}(g) \subseteq f_{n-1}^{\infty}(g)$.

Using Theorem 9 we prove now that the complete network $\left\{g^{N}\right\}$ is a level- $K$ farsightedly stable set for every $K \geq n-1 .^{18}$ Notice that the level of farsightedness needed to sustain the complete network $\left\{g^{N}\right\}$ is quite small when compared to the number of potential networks and the maximum length of paths.

Theorem 12. For criminal networks it holds that $\left\{g^{N}\right\}$ is a level-K farsightedly stable set for every $K \geq n-1$.

Proof. By Lemma 3 we have that $g^{N} \in D_{1}$. By Lemma 4 we have that for every $g^{\prime} \in \mathbb{G} \backslash\left\{g^{N}\right\}$ it holds that $g^{N} \in f_{n-1}^{\infty}\left(g^{\prime}\right) \subseteq f_{K}^{\infty}\left(g^{\prime}\right)$, where the inclusion follows from Lemma 2. We are now in a position to apply Theorem 9 and conclude that $\left\{g^{N}\right\}$ is a level- $K$ farsightedly stable set.

\footnotetext{
${ }^{18}$ Herings, Mauleon and Vannetelbosch (2009) show that in the example of criminal networks with $n$ players, the complete network $\left\{g^{N}\right\}$ is a pairwise farsightedly stable set.
} 
How about the uniqueness of $\left\{g^{N}\right\}$ as a level- $K$ farsightedly stable set? It is tempting to use the approach of Theorem 9 and show such a result by proving that $g^{N} \in P_{K}$. However, consider the case with 6 players and let $g^{\prime}=g^{N}-16-26-35-45$. For any value of $B$ and $\phi,{ }^{19}$ we claim that $g^{\prime} \in f_{12}\left(g^{N}\right)$, so $g^{N} \notin P_{12}$. Since the network $g^{\prime}$ is connected, $d_{1}\left(g^{\prime}\right)=d_{2}\left(g^{\prime}\right)=d_{3}\left(g^{\prime}\right)=d_{4}\left(g^{\prime}\right)=4$, and $d_{5}\left(g^{\prime}\right)=d_{6}\left(g^{\prime}\right)=$ 3 , it holds for any $i \in\{1,2,3,4\}$ that $Y_{i}\left(g^{\prime}\right)=(1 / 4-\phi / 24) B>B / 6=Y_{i}\left(g^{N}\right)$ and for any $j \in\{5,6\}$ that $Y_{j}\left(g^{\prime}\right)=0<B / 6=Y_{j}\left(g^{N}\right)$. The construction of the farsighted improving path is, however, more subtle than simply deleting the links $16,26,35$, and 45 in some order. Indeed, after the deletion of three such links, there are exactly two players with the maximum degree and they would get strictly lower payoffs by cutting their link, and would be unwilling to do so. The way to avoid this problem requires more farsightedness and involves all players in $\{1,2,3,4\}$ first cutting two of their mutual links, before severing the links with players 5 and 6 , and finally restoring their mutual links. One explicit farsighted improving path results from $g^{N}-12-23-34-41-16-26-35-45+12+23+34+41$ and takes 12 steps. We have denoted the player with an incentive to cut a link first, so -16 for instance means that Player 1 cuts his link with Player 6, whereas -61 would mean that Player 6 cuts his link with Player 1. It can be verified that each step in this farsighted improving path is feasible indeed.

We conclude this section by showing that if players are not too farsighted, then $g^{N} \in P_{K}$, so $\left\{g^{N}\right\}$ is the unique level- $K$ farsightedly stable set. More precisely, we will from now on consider $K=n-1$. We show first that any network in $f_{n-1}\left(g^{N}\right)$ has a single component involving all players.

Lemma 5. For criminal networks it holds for every $g^{\prime} \in f_{n-1}\left(g^{N}\right)$ that $P\left(g^{\prime}\right)=\{N\}$.

Proof. Consider the criminal group $S$ of Player 1 in $g^{\prime}$. We show that it contains all players. Suppose it contains only $s \leq n-1$ players. Then, starting from $g^{N}$, those $s$ players have to cut all their links with all other players in $N \backslash S$. This involves at least $s(n-s)$ steps. For fixed $n$, the concavity of $s(n-s)$ in $s$ implies that it is minimized at $s=1$ or $s=n-1$. Substitution of these values of $s$ shows the minimum to be equal to $n-1$ at both $s=1$ and $s=n-1$. When the $s$ players cut all their links with all other players in $N \backslash S$, all the players in $N$ are strictly worse off, since the probability of being caught has strictly increased and the probability

\footnotetext{
${ }^{19}$ We maintain the assumption that $\phi<n /(n-1)$.
} 
of winning the loot has decreased, contradicting $g^{\prime} \in f_{n-1}\left(g^{N}\right)$.

We show next that the complete network $g^{N}$ is level- $(n-1)$ pairwise stable.

Lemma 6. For criminal networks it holds that $g^{N} \in P_{n-1}$.

Proof. Suppose $g^{\prime}$ is an element of $\tilde{f}_{n-1}\left(g^{N}\right)$. Let $g_{0}, \ldots, g_{K}$ with $g_{0}=g^{N}$ and $g_{K}=g^{\prime}$ be a farsighted improving path of length $K \leq n-1$. By Lemma 5 it holds that $c_{i}\left(g^{\prime}\right)$ is independent from $i$, so we denote it by $c$. Let $M \subseteq N$ be such that $i \in M$ if and only if $d_{i}\left(g^{\prime}\right)=c$ and denote the cardinality of $M$ by $m$. It cannot be that $m=n$, since then all players have lower payoffs in $g^{\prime}$ than in $g^{N}$ because the probability of being caught is higher in $g^{\prime}$ than in $g^{N}$. Since $g^{\prime}$ is connected by Lemma 5, it follows that $Y_{j}\left(g^{\prime}\right)=0$ for all $j \in N \backslash M$. A player $j \in N \backslash M$ will therefore not sever a link at any network in the farsighted improving path $g_{0}, \ldots, g_{K}$. It follows that

$$
\sum_{i \in M}\left(n-1-d_{i}\left(g^{\prime}\right)\right) \geq \sum_{j \in N \backslash M}\left(n-1-d_{j}\left(g^{\prime}\right)\right) .
$$

Since $d_{i}\left(g^{\prime}\right)>d_{j}\left(g^{\prime}\right)$ whenever $i \in M$ and $j \in N \backslash M$, we have that $m>n / 2$.

Since at least one link $i j$ with $i \in M$ and $j \in N$ is missing in $g^{\prime}$, it follows that the maximum degree in $g^{\prime}$ satisfies $c \leq n-2$.

The number $K$ is equal to the number of times a link $i j$ is severed with $i \in M$ and $j \in N \backslash M$ plus the number of times a link $i j$ is cut with $i, j \in M$ plus the number of link additions. We argue next that lower bounds for these three numbers are given by $2(n-m), 2 m-n-1$, and 1 , respectively.

Since all players in $N \backslash M$ experienced the severance of at least two links, and any such link is cut by a player in $M$, a lower bound for the first number is $2(n-m)$.

For $k=0, \ldots, K$, let $L\left(g_{k}\right)=\left\{i \in N \mid d_{i}\left(g_{k}\right)=n-1\right\}$ be the set of players with degree $n-1$ and let $\ell\left(g_{k}\right)=\# L\left(g_{k}\right)$ be its cardinality. Clearly, it holds that $\ell\left(g^{N}\right)=n$ and $\ell\left(g^{\prime}\right)=0$. Let $k^{\prime}$ be the lowest value of $k$ such that $\ell\left(g_{k}\right) \leq m$ for all $k \geq k^{\prime}$. Since $\ell\left(g_{k}\right)-\ell\left(g_{k+1}\right) \leq 2$, we find that $\ell\left(g_{k^{\prime}}\right)=m$ or $\ell\left(g_{k^{\prime}}\right)=m-1$. The sum of the cardinality $\ell\left(g_{k^{\prime}}\right)$ of $L\left(g_{k^{\prime}}\right)$ and the cardinality $m$ of $M$ is therefore at least $2 m-1$. Since there are only $n$ players, it follows that $\#\left(L\left(g_{k^{\prime}}\right) \cap M\right)$, the cardinality of the set of players in $L\left(g_{k^{\prime}}\right)$ that belong to $M$, is at least $2 m-n-1$.

For all $k \geq k^{\prime}$, for all $i \in L\left(g_{k}\right)$, it holds that $Y_{i}\left(g_{k}\right)>Y_{i}\left(g^{\prime}\right)$, since the loot has to be shared with less or the same number of criminals and the probability of 
being caught is strictly less when comparing $g_{k}$ to $g^{\prime}$. Such a player $i$ will therefore never choose to sever a link himself, so whenever a link involving player $i \in L\left(g_{k}\right)$ is severed when going from $g_{k}$ to $g_{k+1}$, it must be by a player in $M \backslash L\left(g_{k}\right)$. It follows that $\ell\left(g_{k}\right)-\ell\left(g_{k+1}\right) \leq 1$. Since $\#\left(L\left(g_{k^{\prime}}\right) \cap M\right) \geq 2 m-n-1$, we find that going from $g_{k^{\prime}}$ to $g^{\prime}$ involves the deletion of at least $2 m-n-1$ links $i j$ with $i, j \in M$.

We argue next that the move from $g_{K-1}$ to $g_{K}$ involves a link addition. Suppose not, then there is $i j$ with $i \in M$ such that $g_{K}=g_{K-1}-i j$ and $Y_{i}\left(g_{K}\right)>Y_{i}\left(g_{K-1}\right)$. Since $d_{i}\left(g_{K-1}\right)=c_{i}\left(g_{K-1}\right)>c_{i}\left(g_{K}\right)=d_{i}\left(g_{K}\right)$, it follows that at $g_{K}$, $i$ has to share the loot with more criminals and has a higher probability of being caught than at $g_{K-1}$, so $Y_{i}\left(g_{K}\right)<Y_{i}\left(g_{K-1}\right)$, leading to a contradiction. Consequently, the move from $g_{K-1}$ to $g_{K}$ involves a link addition.

We have proved that $K \geq 2(n-m)+2 m-n-1+1=n$, which contradicts our original supposition that $K \leq n-1$. Consequently, it holds that $\tilde{f}_{n-1}\left(g^{N}\right)=\emptyset$.

Using Theorem 9 we prove now that the complete network $\left\{g^{N}\right\}$ is the unique level- $(n-1)$ farsightedly stable set.

Theorem 13. For criminal networks it holds that $\left\{g^{N}\right\}$ is the unique level- $(n-1)$ farsightedly stable set.

Proof. By Lemma 3 we have that $g^{N} \in D_{1}$. By Lemma 4 we have that for every $g^{\prime} \in \mathbb{G} \backslash\left\{g^{N}\right\}$ it holds that $g^{N} \in f_{n-1}^{\infty}\left(g^{\prime}\right)$. By Lemma 6 it holds that $g^{N} \in P_{n-1}$. We are now in a position to apply Theorem 9 and conclude that $\left\{g^{N}\right\}$ is the unique level- $(n-1)$ farsightedly stable set.

Structural properties of criminal networks must be taken into account to better understand the impact of peer influence on delinquent behavior and to address adequate and novel delinquency-reducing policies. Hence, it is important to acquire knowledge about the level of farsightedness of criminals to determine which criminal networks are likely to emerge in the long run. ${ }^{20}$

\footnotetext{
${ }^{20}$ Ballester, Calvo-Armengol and Zenou (2010) develop a criminal network game where each delinquent decides how much delinquency effort to exert. The network is determined endogenously by allowing players to join the labor market instead of committing criminal activities. They find that the optimal enforcement policy consists of removing some key player or some key group. Such a policy is complex since it depends both on the wage and on the network. Indeed, the removal of some players may induce further voluntary moves of other players who now find it profitable to leave their criminal activities and join the labor market.
} 


\section{Conclusion}

We study the stability of social and economic networks when players are limited farsighted. Pairwise stability is a very important tool in network analysis. One shortcoming of pairwise stability is the lack of farsightedness. Players do not anticipate that other players may react to their changes. However, farsighted stability often requires too much foresight on behalf of the players. Hence we propose an intermediate concept, namely level- $K$ farsighted stability, that can be used to study the influence of the degree of farsightedness on network stability.

In the present paper, we assume that all players have a reasoning horizon of length $K$, where $K$ can be any natural number. By doing so we cover myopic and full farsighted behavior as extreme cases, and we are able to provide a tractable concept to study the influence of the degree of farsightedness on the predictions of network formation models. Recently, Kirchsteiger, Mantovani, Mauleon and Vannetelbosch (2013) show experimental evidence that suggests that subjects are consistent with intermediate levels of farsightedness.

We think that our concept of level- $K$ farsighted stability could be useful in the study of the level of farsightedness needed in order to sustain certain networks as stable outcomes. Consider for instance the formation of a network of bilateral free trade agreements. Goyal and Joshi (2006) show that global free trade, represented by the complete network, is pairwise stable, implying that global free trade, if reached, will prevail. However, the complete network is not the unique pairwise stable network and Goyal and Joshi's analysis leaves out the issue whether global free trade can be obtained from the empty network or any preexisting free trade network when countries are myopic.

Recently, Zhang, Xue and Zu (2013) complements the analysis of Goyal and Joshi (2006) by examining whether global free trade can result from a sequence of bilateral free trade agreements when countries are farsighted. They show that the complete network constitutes a pairwise farsightedly stable set, though is not uniquely so. In particular, they show that there is a farsighted improving path from the empty network to the complete network which involves link additions only, while farsighted improving paths from preexisting free trade networks may involve both link deletion and addition. However, in order to obtain this result, Zhang, Xue and $\mathrm{Zu}$ (2013) assumes that countries have perfect foresight. Could global free trade 
result from a sequence of bilateral free trade agreements when countries are neither myopic nor completely farsighted but have some limited degree of farsightedness? Which is the level of farsightedness needed in order to reach the global free trade network from any preexisting free trade network? Structural properties of free trade networks must be taken into account to better understand whether free trade areas are conductive or detrimental to globalization. Hence, it is important to acquire knowledge about the level of farsightedness of countries to determine which free trade networks are likely to emerge in the long run and the kind of intervention that supranational authorities should undertake in order to promote global free trade.

One-to-one matching problems are another simple example of network formation. Agents are either male or female, and a man (woman) only wants to be matched to a woman (man) or to him(her)self. For one-to-one matching problems, Ehlers (1997) characterizes the von Neumann-Morgenstern (vNM) stable set using a direct dominance relation and shows that this set can be larger than the core. Mauleon, Vergote and Vannetelbosch (2011) provides the characterization of vNM farsightedly stable sets (that are also pairwise farsightedly stable sets of Herings, Mauleon and Vannetelbosch, 2009): a set of matchings is a vNM farsightedly stable set if and only if it is a singleton subset of the core. Thus, contrary to the vNM (myopically) stable sets, vNM farsightedly stable sets cannot include matchings that are not in the core. However, to obtain this result, Mauleon, Vergote and Vannetelbosch (2011) assumes that agents are completely farsighted. When this is the case, each core element can be reached by means of a farsighted improving path from any other matching. How robust is this result to the introduction of limited farsightedness? Which is the level of farsightedness that guarantee that only core elements are stable in the long run?

Empirical work suggests that R\&D collaboration between firms is common. Typically, collaboration ties are bilateral and are embedded within a broader network of similar ties with other firms. Several recent contributions have examined the incentives of firms to collaborate with each other in order to reduce its marginal cost of production (see, for instance, Goyal and Moraga-Gonzalez (2001) and Goyal and Joshi (2003)). All these contributions differ in the way they model the reduction in marginal cost due to the $R \& D$ collaborative agreement as well as the spillovers on competitors, but they all assume that firms are myopic. Recently, Mauleon, Sempere-Monerris and Vannetelbosch (2014) studies the R\&D networks that would arise in the long run, analyzing both the case with myopic firms and the one with 
farsighted firms. The reduction of the marginal cost of production for one firm is assumed to be proportional to the number of firms it is connected to. They show that firms form networks consisting of two components, with firms in the largest component obtaining a competitive advantage upon the other group. The difference in the number of firms between the two components is at most three if firms are myopic. However, if firms are farsighted, the largest component comprises roughly three-quarters of the firms, hence the larger group of firms can derive a much greater competitive advantage from $R \& D$ collaborations relative to the other group. In order to give the correct incentives for firms to form collaborative agreements, it is necessary to know the structural properties of the R\&D networks that will emerge in the long run. For this reason, and given the different predictions about the stable $R \& D$ networks when firms are either myopic or farsighted, it is crucial to know which R\&D networks firms with intermediate degrees of farsightedness would form. Which is the level of farsightedness needed to obtain the predictions for completely farsighted firms? How do the predictions about stable R\&D networks relate to the degree of farsightedness? We argue here that our concept could be used as a first step in trying to answer all these questions.

\section{Acknowledgments}

Vincent Vannetelbosch and Ana Mauleon are Senior Research Associates of the National Fund for Scientific Research (FNRS). Financial support from the Spanish Ministry of Economy and Competition under the project ECO2012-35820 is gratefully acknowledged.

\section{References}

[1] Aumann, R. and R. Myerson, 1988. Endogenous formation of links between players and coalitions: An application of the Shapley value. In Roth. A. (Ed.). The Shapley value. Cambridge University Press, Cambridge. pp. 175-191.

[2] Ballester, C., A. Calvo-Armengol and Y. Zenou, 2010. Delinquent networks. Journal of the European Economic Association 8, 34-61.

[3] Calvo-Armengol, A. and Y. Zenou, 2004. Social networks and crime decisions: the role of social structure in facilitating delinquent behavior. International Economic Review 45, 939-958. 
[4] Camerer, C.F., T.-H. Ho and J.-K. Chong, 2004. A cognitive hierarchy model of games. Quarterly Journal of Economics 119, 861-898.

[5] Chwe, M.S., 1994. Farsighted coalitional stability. Journal of Economic Theory 63, 299-325.

[6] Costa-Gomes, M.A., V.P. Crawford and B. Broseta, 2001. Cognition and behavior in normal-form games: an experimental study. Econometrica 69, 1193-1235.

[7] Crawford, V.P., M.A. Costa-Gomes and N. Iriberri, 2013. Structural models of nonequilibrium strategic thinking: theory, evidence, and applications. Journal of Economic Literature 51, 5-62.

[8] Dutta, B., S. Ghosal and D. Ray, 2005. Farsighted network formation. Journal of Economic Theory 122, 143-164.

[9] Dutta, B. and S. Mutuswami, 1997. Stable networks. Journal of Economic Theory $76,322-344$.

[10] Ehlers, L., 2007. Von Neumann-Morgenstern stable sets in matching problems. Journal of Economic Theory 134, 537-547.

[11] Goyal, S., and J.L. Moraga-Gonzalez, 2001. R\&D networks, RAND Journal of Economics 32, 686-707.

[12] Goyal, S., and S. Joshi, 2003. Networks of collaboration in oligopoly, Games and Economic Behavior 43, 57-85.

[13] Goyal, S. and S. Joshi, 2006. Bilateralism and free trade. International Economic Review 47, 749-778.

[14] Herings, P.J.J., A. Mauleon and V. Vannetelbosch, 2004. Rationalizability for social environments. Games and Economic Behavior 49, 135-156.

[15] Herings, P.J.J., A. Mauleon and V. Vannetelbosch, 2009. Farsightedly stable networks. Games and Economic Behavior 67, 526-541.

[16] Jackson, M.O., 2008. Social and economic networks. Princeton University Press: Princeton, NJ, USA. 
[17] Jackson, M.O. and A. van den Nouweland, 2005. Strongly stable networks. Games and Economic Behavior 51, 420-444.

[18] Jackson, M.O. and A. Watts, 2002. The evolution of social and economic networks. Journal of Economic Theory 106, 265-295.

[19] Jackson, M.O. and A. Wolinsky, 1996. A strategic model of social and economic networks. Journal of Economic Theory 71, 44-74.

[20] Kirchsteiger, G., M. Mantovani, A. Mauleon and V. Vannetelbosch, 2013. Limited farsightedness in network formation. CORE Discussion Paper 2013-33. Université catholique de Louvain, Belgium.

[21] Krishnan, P. and E. Sciubba, 2009. Links and architecture in village networks. Economic Journal 119, 917-949.

[22] Mauleon, A., J.J. Sempere-Monerris and V. Vannetelbosch, 2014. Farsighted R\&D networks. mimeo.

[23] Mauleon, A. and V. Vannetelbosch, 2004. Farsightedness and cautiousness in coalition formation games with positive spillovers. Theory and Decision 56, 291-324.

[24] Mauleon, A., V. Vannetelbosch and W. Vergote, 2011. von Neumann Morgernstern farsightedly stable sets in two-sided matching. Theoretical Economics 6, 499-521.

[25] McKelvey, R.D. and T.R. Palfrey, 1992. An experimental study of the centipede game. Econometrica 60, 803-836.

[26] Morbitzer, D., V. Buskens and S. Rosenkranz, 2011. How farsightedness affects network formation. ISCORE Discussion Paper 282. Utrecht University, The Netherlands.

[27] Myerson, R., 1991. Game theory: Analysis of conflict. Harvard University Press: Cambridge, MA, USA.

[28] Nagel, R., 1995. Unraveling in guessing games: an experimental study. American Economic Review 85, 1313-1326. 
[29] Page, F.H., Jr., M. Wooders and S. Kamat, 2005. Networks and farsighted stability. Journal of Economic Theory 120, 257-269.

[30] Page, F.H., Jr. and M. Wooders, 2009. Strategic basins of attraction, the path dominance core, and network formation games. Games and Economic Behavior $66,462-487$.

[31] Patacchini, E. and Y. Zenou, 2008. The strength of weak ties in crime. European Economic Review 52, 209-236.

[32] Ray, D. and R. Vohra, 2013. The farsighted stable set. Working Paper 2013-11. Brown University, USA.

[33] Stahl, D.O. and P. Wilson, 1994. Experimental evidence on players' models of other players. Journal of Economic Behavior and Organization 25, 309-327.

[34] Xue, L., 1998. Coalitional stability under perfect foresight. Economic Theory $11,603-627$.

[35] Zhang, J., L. Xue and L. Zu, 2013. Farsighted free trade networks. Intenational Journal of Game Theory 42, 375-398. 